\title{
Stimulus dependence of directed information exchange between cortical layers in macaque $\mathrm{V} 1$
}

1 Marc Alwin Gieselmann ${ }^{1}$, Alexander Thiele ${ }^{1 *}$

$2 \quad{ }^{1}$ Biosciences Institute, Newcastle University, UK

4 * Corresponding and senior author: Alexander Thiele, Biosciences Institute, Newcastle University, 5 Newcastle upon Tyne, NE2 4HH. E-mail: alex.thiele@ncl.ac.uk, Orcid ID: https://orcid.org/0000-0003$6 \quad \underline{4894-0213}$

\section{Summary}

8 Perception and cognition require the integration of feed-forward sensory information with feedback

9 signals. Feed-forward and feedback signals have been associated with specific cortical laminae and 10 specific oscillatory frequencies. We used stimuli of different sizes to isolate spectral signatures of 11 feedforward and feedback signals, and their effect on communication between layers in primary 12 visual cortex of male macaque monkeys. Small stimuli, centered on the neurons' receptive fields 13 elicited broad band gamma frequency oscillations predominantly in the superficial layers. These 14 Granger causal originated in upper layer 4 and lower supragranular layers, in line with existing feed15 forward models of oscillatory responses. However, large stimuli extending into the far surround of 16 neurons' receptive fields, generated strong narrow band gamma oscillatory activity across cortical 17 layers. They Granger causal arose in layer 5, were conveyed through layer 6 to superficial layers, and 18 violated existing models of feedback spectral signatures. Equally surprising, with large stimuli, alpha 19 band oscillatory activity arose predominantly in granular and supragranular layers, and 20 communicated in a feed-forward direction. This shows that oscillations in specific frequency bands 21 can be dynamically modulated to serve feedback and feed-forward communication and are not 22 restricted to specific cortical layers in V1. 
Introduction

28 The multiple layers of the neocortex show an elaborate connectivity pattern, which predicts a specific directionality of interlaminar communication (Callaway, 1998; Douglas and Martin, 2004; Douglas et al., 1989). Feedforward inputs predominantly terminate in layer 4 of sensory cortical areas, before being passed to layers $2 / 3$ and then onwards to layers $5 / 6$, where recurrent inputs to layer $2 / 3$ arise (Callaway, 1998; Callaway, 2004). Conversely, feedback connections predominantly terminate in layer 1 and 5 (Rockland and Pandya, 1979), from where the information is parsed to layers 2/3. Local field potential (LFP) recordings, using different stimulus and task conditions, have provided physiological support for these anatomical pathways. Current source density analysis of the LFP, as well as latency of spiking activity has demonstrated that the earliest activity elicited by stimuli centered on the classical receptive field occurs in layer 4C (Bijanzadeh et al., 2018). Stimuli restricted to the far receptive field surround, which are hypothesized to affect processing in V1 primarily through feedback projections (Bair et al., 2003), initially activate V1 in upper superficial layers and in layer 5 (Bijanzadeh et al., 2018). In addition to this anatomical segregation of feed-forward and feedback signal input to V1, it has been proposed that feedforward and feedback signals have separable spectral signatures of the LFP. Feedback processing has been aligned with low frequency (theta, alpha band) oscillatory activity, dominating activity in infragranular layers (Buffalo et al., 2011; van Kerkoerle et al., 2014), and with beta band oscillations (Bastos et al., 2015a; Xing et al., 2012). Conversely, feedforward processing has been associated with higher frequency oscillatory activity (gamma band), originating in layer 4, dominating in (or even being largely restricted to) supragranular layers (Bastos et al., 2015a; Bollimunta et al., 2011; Buffalo et al., 2004; Buffalo et al.,

48 2011; Maier et al., 2010; Spaak et al., 2012; van Kerkoerle et al., 2014; Xing et al., 2012). However, the generality of this scenario has recently been questioned (Haegens et al., 2015), whereby the strongest low frequency (alpha) oscillations generators were found in supragranular layers of sensory cortex. Moreover, while most studies failed to find prominent gamma oscillations outside supragranular layers (Buffalo et al., 2011; Maier et al., 2010; Xing et al., 2012), exceptions do exist

53 (Maier et al., 2011).

54 To address these controversies and investigate the origin and spectral signature of feed-forward and feedback signals in detail we recorded across cortical layers of V1 of awake macaque monkeys, while manipulating stimulus size systematically. Increasing the stimulus size, invokes center surround interactions, which result in specific input patterns across a cortical column (Bijanzadeh et al., 2018), and which strongly alter the spectral signatures LFPs. Increasing the size of a patch of an orientated grating centered on a receptive field (RF) increases gamma power in conjunction with a general decrease of neurons firing rate (Bauer et al., 1995; Gieselmann and Thiele, 2008; Jia et al., 2011; Ray 
61 and Maunsell, 2010). If this increased gamma power was a signature of a feedforward signal, then

62 we would expect it to arise in either layer 4, or layer $2 / 3$ of V1. Conversely, the feedback signals

63 should show spectral signatures in lower frequency bands, such as alpha (van Kerkoerle et al., 2014),

64 which should arise and dominate in layers 1 and 5 (Bastos et al., 2015a; van Kerkoerle et al., 2014).

65 While our results support some of the features outlined above, they violate them in multiple aspects,

66 and show that feed-forward and feedback signals attain stimulus dependent oscillatory frequencies

67 across cortical layers on V1.

\section{Results}

70 We recorded neuronal activity from area V1 from 16 contact laminar electrodes in two passively viewing male macaque monkeys. Electrodes were inserted perpendicular to the cortical surface, aiming to record from within a single V1 cortical column. To ensure consistency of our analysis we rejected experiments from further analysis if the current-source-density (CSD, see methods) profiles did not meet any of the following three conditions: (1) An identifiable current source as early as 50 ms after the stimulus onset. (2) The "early" current source is identified on a channel between the seventh and twelfth contact (from the top of the electrode array, to ensure a minimum coverage of cortical layers 2-6). (3) A pattern of the CSD-profile that is consistent with the majority of experiments. After applying the rejection criteria we were left with 24 experiments (monkey 1: $\mathrm{n}=17$, monkey 2: $\mathrm{n}=7$ ) from 3 hemispheres which were included into this study.

81 Figure 1 shows the alignment of neuronal data from an example experiment. The data were obtained 82 during receptive field (RF) mapping (Figure 1B) and during presentation of gratings (Figure 1C,D). In this recording the eighth channel from the top was defined as the alignment channel indicating putative layer IVc at zero depth (Figure 1A). Correspondingly, the RF maps of the envelope of multiunit-activity (MUAe, see methods) showed the early emergence of a visual RF in the same channel between 30-50 ms (Figure 1B left), demonstrating that the early current sink coincides with the earliest activation from thalamo-cortical afferents. This electrode placement resulted in contacts in supragranular layers (SG), alignment layer IVc, and infragranular layers (IG) (Figure 1A). The gray matter region (from third channel from top to sixths from the bottom in Figure 1A) is flanked by two large current sources at the top and at the bottom (Figure 1D). The perpendicularity of probe orientation relative to the cortical surface is indicated by the good spatial alignment of RF-centers (Figure 1B). The LFP responses in the gray matter region show strong evoked potentials, and sustained oscillations (Figure 1C). The lower five channels in Figure 1 were located in the white matter, and possibly in area V2, as evident by a sudden shift in visual RF's (Figure 1B, lowest 
contacts). While the strength of the LFP-signal seems to be unchanged (Figure 1C) for these contacts, current sources and sinks were weaker and less structured than in the gray matter.

98 We next focus our analysis on basic phenomena of spiking activity and current source density changes

99 with different sized stimuli, as these provide insight into the underlying pathways that mediate 100 changes (feed-forward, lateral, and feedback connections), as well as providing a benchmark against 101 previously published results.

\section{Response latency as a function of cortical depth and stimulus size}

104 We calculated spiking latencies by using the MUAe signal using a curve-fitting procedure (figure 2A, 105 see methods and (Self et al., 2013)). The shortest latencies occurred in granular layers, followed by 106 infragranular layers, with supragranular layers generally lagging behind (figure 2B). This overall 107 pattern was independent of stimulus size used. However, stimulus size altered response latencies in 108 granular and in infragranular layers (figure 2B). In granular layers larger stimuli resulted in up to $109 \sim 3 \mathrm{~ms}$ longer latencies ( $\mathrm{p}<0.01$, ANOVA, figure $2 \mathrm{C}$ ), while in infragranular layers they caused up to $110 \sim 6 \mathrm{~ms}$ shorter latencies ( $\mathrm{p}<0.001$, ANOVA, figure 2C). Thus, when large stimuli were presented the 111 lag between granular and infragranular layers was reduced to $\sim 2 \mathrm{~ms}$ (from $\sim 7 \mathrm{~ms}$ for small stimuli, 112 figure 2C). No significant changes were found for supragranular layers $(p=0.59$, one-way ANOVA, 113 Figure 2C). Latency changes also appeared to occur in the top-most layer, although this was not 114 significant, possibly because of relatively small sample sizes (due to electrode placement not every 115 recording included that layer/contact).

116 The CSD profile equally changed with stimulus size. Figure 2D and E show the average CSD profile 117 across all experiments for a small $\left(0.5^{\circ}\right)$ and a large $\left(15.0^{\circ}\right)$ stimulus. The CSD-profile of each 118 experiment was normalized to the peak of the early onset current sink in the alignment layer $(0 \mu \mathrm{m})$. 119 Figure 2E shows a surface plot of time depth CSD differences plot (difference between CSD profiles 120 in $\mathrm{C}$ and $\mathrm{D}$, where only significant differences are displayed ( $\mathrm{p}<0.05$, cluster-mass-test). In granular 121 and supragranular layers we observed moderate decreases in inward current amplitudes with larger 122 stimuli, which occurred as early as $50 \mathrm{~ms}$ in granular layers. Most of the changes in and above layer 1230 can be described as a general attenuation of current amplitudes. In other words, the overall laminar 124 pattern of current sinks and sources in granular and supragranular layers was similar in the CSD 125 profile of small and large stimuli, but it was overall slightly attenuated for large stimuli, probably due 126 to surround suppression effects at the level of the lateral-geniculate-nucleus (LGN). The currents 127 (sinks) in infragranular layers were also mostly reduced, but in addition changes in the pattern of sinks and sources occurred. Specifically a current sink emerged after at $\sim 75 \mathrm{~ms}$ after stimulus onset 
at $-600 \mu \mathrm{m}$ (figure $2 \mathrm{D}, \mathrm{E}$ ), where a source was present when small stimuli were presented. This indicates that stimulating the surround of a V1 RF results in additional input to infragranular layers.

132 We next focused on surround suppression/enhancement effects on MUAe, which are most profound 133 during later parts of the response. Here we analyzed the activity from 200 to $500 \mathrm{~ms}$ after stimulus 134 onset. Surround suppression affected all cortical layers. However, it was more pronounced in supragranular and granular than in infragranular layers (figure 3A,B). Moreover, the stimulus size that resulted in maximal activity equally varied between layers, with slightly larger stimuli $(0.75 \mathrm{deg}$, $1.5 \mathrm{deg})$ eliciting maximal activity in infragranular layers, while the smallest stimulus (0.5 deg) elicited maximal activity in supragranular layers (figure 3C).

140 Together the results from figures 2 and 3 are compatible with recent reports suggesting that stimuli 141 extending into the far surround of neurons invoke center surround interactions through feedback 142 connections from higher cortical areas that terminate predominantly in layers 1 and 5 (Bijanzadeh et 143 al., 2018; Nassi et al., 2013; Nurminen et al., 2018; van Kerkoerle et al., 2014).

\section{LFP spectral power and phase relationship analysis}

147 We now turn to the question how feedback signals impose their effects on local cortical processing 148 in area V1. It has been argued that feedback signals can be characterized in terms of low frequency 149 (alpha, beta) oscillatory activity, while feedforward signals can be characterized in terms of theta and 150 gamma frequency oscillations (Bastos et al., 2015a; Bosman et al., 2012; Buschman and Miller, 2009; 151 Fries, 2015). A simple prediction would thus be that all stimulus sizes elicit gamma frequency oscillations in layers $2 / 3$, as all of the stimuli will invoke feedforward drive. Within this context, two scenarios are possible. Firstly, gamma frequency oscillations in L2/3 are either slightly reduced when

154 larger stimuli are presented, due to the effects of surround suppression that are inherited from the 155 LGN. Alternatively, their spectral power increases as stimulus size increases, because large stimuli 156 invoke stronger inhibitory surround interactions, which through pyramidal-inhibitory interactions 157 drive gamma frequency oscillations (Tiesinga and Sejnowski, 2009). Additionally, infragranular 158 layers should show limited gamma frequency power (Dougherty et al., 2017; Maier et al., 2011; Spaak 159 et al., 2012; Xing et al., 2012), but they should show increased low frequency (alpha/beta) oscillations 160 with increasing stimulus size as the level of feedback input increases (Bastos et al., 2015a; Bosman 161 et al., 2012; Buffalo et al., 2011; van Kerkoerle et al., 2014). 
164 (LFPbp, methods). This was necessary, as the raw LFP is not a sufficiently local signal to allow for

165

166

167

168

169

170

171

172

173

174

175

176

177

178

179

180

181

182

183

184 We quantified the effects of stimulus size on spectral power by calculating the mean power of the

185 LFPbp-signal in discrete frequency bands, normalized (z-score) to the spontaneous period $300 \mathrm{~ms}$

186 before stimulus onset (methods). This was done for the theta ( $\theta$-band, 4-8 Hz, fig. 5A-C), alpha ( $\alpha$ band, $8-13 \mathrm{~Hz}$, fig. 5D-F), beta ( $\beta$-band, $14-25 \mathrm{~Hz}$, fig. 5G-I) and low gamma ( $\gamma$-band, 35-55 Hz, fig. $5 \mathrm{~J}-\mathrm{L}$ ) range. Figure $5 \mathrm{~A}, \mathrm{D}, \mathrm{G}, \mathrm{J}$ show the average depth profiles of band power in response to a small (0.5deg, blue) and a large (15deg, red) stimulus. For all frequency bands, stimulus size modulated the band, but these modulations differed between layers and frequency bands. For the $\theta$-band, $\alpha$-band and $\beta$-band, stimulus size induced changes are most pronounced for granular and lower supragranular layers (Figure 5C,F,I). For infragranular layers, larger stimuli caused a reduction in $\theta$-band (Figure 5B), no change in $\alpha$-band or $\beta$-band, and strong increases in $\gamma$-band. Overall, larger stimuli caused significantly more $\gamma$-band across all contacts (Fig. 5J), but relative changes are strongest in supragranular and infragranular layers (Figure 5L). 


\section{Gamma-connectivity across cortical laminae}

198 Previous studies have argued that phase-amplitude-coupling (PAC) of oscillatory activity can provide 199 insight into the directionality of information flow for a given frequency band. Specifically it was 200 shown that alpha frequency showed a PAC that appeared to originate in infragranular and upper 201 supragranular layers, progressing towards middle supragranular layers (van Kerkoerle et al., 2014).

202 Conversely, gamma frequency PAC originated in granular or lower supragranular layer progressing 203 towards upper supragranular and infragranular layers respectively. To analyze this for different 204 stimulus sizes, we computed the phase-triggered average (PTA) to estimate the phase-amplitude relationship of oscillations between electrode contacts for different frequency bands. Phase-triggeredaveraging describes the alignment of LFPbp signals of a given electrode contact to the phase of an oscillation at a defined frequency at the reference-channel. The aligned LFPbp is then averaged across the number of alignments. Temporal structure in the resulting histogram can be interpreted as a phaseamplitude-coupling between the two signals. Figure 6 shows the depth profiles of the 4 main frequency band PTA-histograms for a reference channel at $-150 \mu \mathrm{m}$, averaged across all experiments.

211 As expected, the depth used as the reference phase of the oscillatory-cycle always showed strong 212 phase amplitude coupling, with close to zero phase delays (Figure 6, blue triangles indicate the 213 reference channel). Many other contacts showed coupling to the reference channel for all frequency 214 bands. However, the exact phase coupling varied between frequency bands and between stimulus 215 sizes. Low frequency (theta, alpha) showed a phase progression from layer 5 to layer 6, and then to 216 granular and supragranular layers for small stimuli. This pattern changed radically for larger stimuli, 217 where phase progression was seen bidirectionally from granular to supragranular and to infragranular 218 layer 5, but also from infragranular layer 6 to layer 5. For gamma frequencies, layer 4 and 6 where 219 largely in phase for small stimuli, but almost $90^{\circ}$ out of phase with layer 5. Some continuous phase progression could be seen from granular towards upper supragranular layers. However, for large stimuli phase progression occurred from layer 5 to layer 6 , onwards to granular layers, and then to supragranular layers. These patterns were not artefacts of selecting a specific reference channel (supplementary figure 1-4).

Phase relationships per se do not yield insight into causal interactions, as phase lags can equally be interpreted as phase advances due to the periodicity of the signal. To gain insight into causality we performed spectral Granger-causality (GC) analysis (supplementary figure 5). We then integrated the GC-Index in the 4 different frequency bands analyzed above $(\theta, \alpha, \beta$, and $\gamma$ frequency) and generated connectivity plots using the net GC (difference between directions) for each connection (figure 7). Hence, figure 7 shows the direction and associated strength of granger-causal influence of the 4 
passed the reverse-granger-test (RGT) to protect against spurious results induced by linear mixed noise (Haufe et al., 2013; Vinck et al., 2015). In supplementary figure 6 A and B we show the same data including the connections which failed RGT and the results of the time reversed GC analysis respectively. Figure 7 shows the analysis of non-conditional GC. Conditional GC produced qualitatively equivalent results, albeit with overall smaller granger causality indices. A striking feature of the directionality of all GCs (irrespective of the frequency bands analyzed) was its dominance in the 'upward' direction, namely towards supragranular layers. This indicates that communication was directed towards the cortico-cortical feed-forward channels in V1. Sizeable GCs in the downward direction were usually restricted to granular to infragranular interactions, and to layer 5 to layer 6 interactions. Downward GCs, were generally more pronounced with larger stimuli. In the upward direction strong $\theta$-GCs originated in layer 6 (lowers contact) affecting predominantly supragranular layers, irrespective of stimulus size. With increasing stimulus size GCs increased in an upward direction between granular and supragranular, and between supragranular-supragranular contacts. Overall a very similar pattern was present for GCs in the $\alpha$ - frequency band. In the $\beta$ frequency band, GCs were fairly weak in the downward direction, when small stimuli were presented,

247 but increased between layer 5 and layer 6 for large stimuli. Upward GCs were pronounced between granular and supragranular, and between supra-supragranular contacts for all stimulus sizes. With increase in stimulus size a pronounced infragranular-supragranular GC occurred. An overall similar pattern was present for $\gamma$-frequency GCs, with an even stronger increase in upwards directed suprasupragranular GCs with stimulus size. The results from figure 7 show that surround modulation results in strongly increased low, mid and high frequency band communication between granular and supragranular compartments, and it results in strongly increased gamma band communication originating from layer 5, which drives gamma frequency changes in layer 6 , and which in turn changes gamma frequency oscillations in upper layer 4 and in layers $2 / 3$.

\section{Discussion}

Using stimuli of different sizes in combination with laminar recordings in primary visual cortex of the macaque revealed that narrow band gamma frequency oscillations occur across cortical layers, and increase across cortical layers with increasing stimulus size. When stimuli encroached on the far surround of the receptive field, the gamma oscillations in layer 5, the feedback recipient layer, exerted pronounced granger causal influences on gamma oscillations in layer 6, which in turn granger causal influenced gamma oscillations in supragranular layers. This pattern of gamma frequency granger causal interactions is compatible with the known anatomical termination sites of feedback connections into V1 (Rockland and Pandya, 1979). The flow of information (granger causal 
interactions in the gamma frequency range), occurred in a manner compatible with the canonical microcircuit, but in a largely reverse direction. Here, information terminating in layer 5, is passed on to layers 6 and 4, who in turn send the information to layers 2/3. However the feedback influence terminating in layer 5 did not show signatures of low frequency oscillations as predicted by recent publications (Bastos et al., 2015a; Bastos et al., 2015b; Bosman et al., 2012; Buffalo et al., 2011;

271 Buschman and Miller; van Kerkoerle et al., 2014). This demonstrates that gamma oscillations are 272 neither restricted to superficial layers, nor are they invariably a signatures of feedforward communication. Moreover, low frequency oscillation GC interactions did not adhere to their proposed role in feedback communication, showing most profound increases in granular to supragranular and supra- to supragranular interactions with stimulus size. This demonstrates that low frequency oscillations are not restricted (or dominant) to infragranular layer, and can attain feedforward roles in 277 V1.

\section{Response latencies and surround suppression as a function of layer and stimulus size}

In line with predictions from the anatomy (Callaway, 1998; Callaway, 2004; Lund, 1988) the earliest responses in V1 occurred in layer $4 \mathrm{C}$, aligned with the earliest current sink. This was followed by responses in layer 6. Responses in supragranular layers were delayed by up to $20 \mathrm{~ms}$. These results qualitatively match previous reports in the anaesthetized macaque (Nowak et al., 1995) and awake monkeys (Self et al., 2013). The early responses in layer 6 are likely mediated by direct inputs from the LGN (Callaway, 1998; Callaway, 2004; Lund, 1988). The relatively faster responses in layer 5 (relative to layer 2/3) are somewhat more surprising, as connections from layer 4C to layer 5 are less dense than those to layer 2/3 (Callaway, 1998). However, the connections to layer 2/3 possibly involve more synapses. Stimulus size affected response latencies in layer 4, where increasing stimulus size initially resulted in decreased latencies, probably due to increased excitatory drive, followed by increased latencies when stimuli were larger than $1.5 \mathrm{deg}$. This increase is most likely driven by surround suppression at the level of the LGN and the retina (Alitto and Usrey, 2008; Bijanzadeh et al., 2018). In layer 5 and 6 increasing stimulus size resulted in shorter response latencies with an asymptote reached once stimuli were $1.5 \mathrm{deg}$ in size. This could be due to a mixture of increased excitation from LGN inputs, and fast feedback inputs (Bair et al., 2002; Bijanzadeh et al., 2018) that compensate for the surround suppression inherited from feedforward inputs when stimuli exceed 1.5 297 deg.

298 Surround suppression equally differed between cortical layers. Surround suppression was largely 299 homogenous for supragranular and granular recording sites, but decreased notably for layers 5 and 6. 300 Both findings are equivalent to previous investigations in the anaesthetized monkey (Sceniak et al., 
2001). We speculate that the decreased surround suppression in layers 5 and 6 is a consequence of the added feedback drive (Angelucci et al., 2002; Bair et al., 2002; Bijanzadeh et al., 2018; Rockland and Pandya, 1979) from higher cortical areas.

\section{Gamma oscillations for different stimulus sizes and layers}

306 In line with previous studies we show that the strength of narrow band gamma frequency oscillations increased monotonically with stimulus size (Bauer et al., 1995; Gieselmann and Thiele, 2008; Jia et al., 2011; Ray and Maunsell, 2010). Gamma frequency oscillations were largely broadband for small stimuli, and most profound in supragranular layers. With increasing stimulus size, gamma frequency oscillations became more narrowband with peak frequencies at $~ 40-50 \mathrm{~Hz}$. With increasing size, power relative to baseline was relatively strong across all cortical layers, including layer 4, even if it was most profound in supra and infragranular layers. These findings contradict previous reports arguing that gamma frequency oscillations are largely confined to supragranular layers (Bollimunta et al., 2011; Buffalo et al., 2011; Maier et al., 2010; Spaak et al., 2012; Xing et al., 2012). However, some studies have reported increased gamma frequency power in granular and infragranular layers upon stimulus onset (Maier et al., 2011; van Kerkoerle et al., 2014). Neither of these have evaluated the effect of stimulus size on gamma frequency power across layers. Van Kerkoerle et al (2014) reported that an increase in alpha frequency power upon structured background presentation in 319 infragranular layers is a dominant feature of the spectral pattern of LFP activity. This increase was relative to the pre-stimulus period, as well as to the condition when a figure was presented in the neurons' receptive field. However, they also saw changes in the gamma frequency, which were stronger for figure conditions, but also present for background conditions. The latter changes were most pronounced in supragranular layer and layer 6, roughly in line with our results.

We found that increasing stimulus size, which invokes strong feedback signals terminating in layers 1 and 5 (Bijanzadeh et al., 2018) resulted in strongly increased narrow band gamma oscillations in layer 5. These in turn Granger causal affected gamma frequency power in layer 6, from where gamma frequency oscillations in layers 4, 3, and 2 were affected. This shows that gamma frequency oscillations are not invariably a signature of feed-forward processing (Bastos et al., 2015a; Bosman et al., 2012; Roberts et al., 2013; van Kerkoerle et al., 2014), but can be an important signature of feedback processing. We do not know whether the input feedback signal itself shows rhythmic activity in the gamma frequency, whether it shows rhythmic activity in a different frequency band, or 333 whether it is rhythmic at all. We speculate that the feedback will show signatures of strong gamma 334 frequency oscillations, and derive its origin from supragranular sources (Markov et al., 2014) of 335 hierarchically close areas, such as V2 or V3 (Markov et al., 2014). However, if there were a cascade 
336 of feedback projections from downstream areas (e.g. V4->V2, V2->V1), each strongly oscillating in

337 the gamma frequency range, then even V2 might show the pattern we describe for V1 here, i.e. strong gamma frequency in infragranular layers. This entails the possibility that the gamma frequency feedback could originate from V2 infra- and supragranular layers. At the same time it has been shown that feedback from hierarchically close areas (such as V2->V1) can predominantly arise in layer 3 and target layer 3 (Markov et al., 2013). This would predict that stimulus size induced changes in granger causal interactions would predominantly occur in layer 3 , if they were triggered from area V2. Long range feedback projections on the other hand terminate in layer 1 and layer 5 (Markov et al., 2013; Rockland and Pandya, 1979). Given the phase relationship between gamma frequency oscillations in different layers and the changes in Granger causal interactions originating in layer 5 with large stimuli, we suggest that a substantial component of stimulus size induced changes in oscillatory activity arises from longer range feedback connections.

At the same time, feedback invoked by stimulus manipulations (i.e. an automatic 'bottom-up' type of feedback) could arise pre-dominantly in hierarchically close areas (e.g. V2), and might be less pronounced from hierarchically distant areas, where lower frequency would dominate due to their infragranular sources (Buffalo et al., 2011; Markov et al., 2014). It is equally possible that this differs from more cognitively driven 'top-down' feedback signatures, which could in principle use a different between our results and those described in van Kerkoerle et. al. (2014), who engaged their animals in a figure ground detection task and an attention demanding curve tracing task. Contrary to van Kerkoerle et. al. (2014), we also did not find that alpha frequency, or other low frequency oscillations, bore the hallmark of feedback signals. They were not restricted to infragranular layers or layer 1 , and alpha frequency based communication within V1, while strongly progressing from layer 6 to supragranular layers for all stimulus sizes, attained prominence within granular and supragranular layers for large stimulus sizes and was directed in an upward direction from there. These results shows that different frequency bands show hallmarks of feed-forward and feedback communication in a flexible, stimulus dependent manner.

We propose that 'cognitive signal' feedback operates between retinotopically aligned neurons (receptive field center aligned), while bottom-up driven feedback operates between retinotopically non-aligned neurons (receptive field center offset). The former could be predominantly excitatory in nature, while the latter might result in net inhibition (even if indirectly). This would explain, why cooling higher areas results in reduced responses in V1 to stimuli restricted to the classical receptive 
371 field, but increased responses to stimuli extending into the receptive field surround (Hupé et al., 372 1998). To clarify whether an automatically (stimulus driven) triggered type of feedback really differs 373 from a 'cognitive' top-down feedback signal, it will be necessary to investigate the layer dependency 374 and the frequency content of interareal interactions under passive viewing conditions and under 375 conditions of active stimulus selection (e.g. spatial attention) demands in future experiments.

Acknowledgements: Supported by the Wellcome Trust (093104), and MRC (MR/P013031/1). We thank the

\section{Methods}

381 Two awake male rhesus monkeys were used for the electrophysiological recordings reported in this study. After initial training, monkeys were implanted with a head holder and recording chambers above V1 under general anesthesia and sterile conditions (for details of surgical procedures, postsurgical analgesics, and general postsurgical treatment, see Thiele et al., 2006). All procedures complied with the European Communities Council Directive RL 2010/63/EC, the U.S. National Institutes of Health Guidelines for the Care and Use of Animals for Experimental Procedures, and the UK Animals Scientific Procedures Act. Animals were motivated to engage in the task through fluid control at levels that do not affect animal physiology and have minimal impact on psychological wellbeing (Gray et al., 2016).

\section{Data acquisition}

392 Recordings were performed using a 16-contact microelectrode (V-probe, Plexon, Dallas, Tex., US). 393 Contacts were arranged vertically along the electrode with an inter-contact spacing of $150 \mu \mathrm{m}$. Probes were lowered into occipital cortex through the intact dura mater by a manually operated hydraulic microdrive system (Narishige International ltd, Japan). Extracellular voltage fluctuations were amplified by means of the Digital Lynx recording system (Neuralynx, Bozeman, Mont., US). The raw signal of each electrode contact was referenced to the guide tube and recorded with 24 bits resolution at a sampling rate of $32 \mathrm{kHz}$. This raw signal was then processed offline to produce two types of signals, the local field potential (LFP) and the envelope of multi-unit activity (MUAe). It is assumed that the LFP reflects the low-frequency changes of post-synaptic potentials (Mitzdorf, 1987), while MUAe captures the aggregate high-frequency spiking activity of the population of neurons around the electrode contact (Super and Roelfsema, 2005). The LFP was obtained by low-pass filtering (IIR-Butterworth, $3^{\text {rd }}$ order, $0.75-300 \mathrm{~Hz}$ ) and down-sampling the raw signal to $\sim 1 \mathrm{kHz}$. To 
405 rectified the raw signal before low-pass filtering at $200 \mathrm{~Hz}$ and down-sampling to $\sim 1$ (Super and 406 Roelfsema, 2005).

\section{Task and Visual stimulation}

408 Experimental timing, behavioral control and stimulus presentation was controlled by Cortex (DOS409 Version 5.95; NIMH) running on IBM-compatible personal computers. The subjects were seated in 410 a primate chair and performed a passive fixation task. A subject started a trial by directing its gaze 411 towards a small fixation spot (red annulus, diameter $1.6^{\circ}$ ) on a CRT-monitor (monkey 1: 1024x768 $412 @ 120 \mathrm{~Hz}$; monkey 2: 1280x1024@100Hz) and received a juice-reward after keeping fixation for 413 another 3-4 s. In each trial we presented a sequence of 3 or 4 (monkey 1 or monkey 2 respectively) 414 grating stimuli centered on the receptive field (RF) of the neuronal column under study.

415 Each grating was presented for $500 \mathrm{~ms}$, preceded by a blank period of $500 \mathrm{~ms}$. Gratings were square416 wave modulated (duty-cycle $=1.5 / \mathrm{deg}$ ) and varied in size between six different diameter $\left(0.5^{\circ}, 0.75^{\circ}\right.$, $4171.5^{\circ}, 3.0^{\circ}, 7.0^{\circ}, 15.0^{\circ}$ ). In monkey 1 the orientation of all gratings was adjusted to the preferred 418 orientation of the neuronal column under study. In monkey 2, we also varied the orientation of the 419 grating between 12 equally spaced orientations.

\section{Experimental protocol}

421 When lowering the electrode, we visually monitored the activity recorded from the probe by means 422 of the recording software. We were able to distinguish cortical LFP activity from the signal recorded 423 above the pial surface by signal amplitude, frequency content and the presence of visually evoked 424 potentials (VEP). In each experiment we initially lowered the electrode to a point in depth where we 425 could identify cortical LFP in the lower 10-12 channels of the probe. We then left the probe in place 426 for a minimum resting period of $30 \mathrm{~min}$ to achieve stable recording conditions. During this resting 427 period cortical LFP activity was usually travelling upwards along the channel array of the probe as a 428 consequence of stress relief on the cortical tissue caused by the penetration. After the resting period 429 we finally adjusted the depth position of the probe such that the two uppermost channels of the array 430 did not show any clear VEPs.

431 We then started estimating the RFs of the cortical column by using a reverse-correlation technique 432 (described in Gieselmann and Thiele, 2008). In short, the RF was mapped by presenting a dark gray 433 square $\left(0.25^{\circ} \times 0.25^{\circ}\right)$ changing positions randomly drawn from a 12 by 9 grid of possible positions 434 every $130 \mathrm{~ms}$. The experimenter then estimated the RF center from the spike probability at each of 435 the grid position. For monkey 1 we estimated the preferred orientation in a similar fashion (described 436 in Gieselmann and Thiele, 2008)..

\section{Extracting the locally generated LFP}

438 The LFP is a sum of a number of locally generated components which are shared across multiple 
449

450

neighboring electrodes due to volume conduction (Mitzdorf and Singer, 1979; Schroeder et al., 1991). To extract the locally generated LFP we transformed the LFP in two ways depending on the two response periods of interest. The early, "phasic" part of the neuronal response (0-200 ms after stimulus onset) contains the VEP, which are transient voltage deflections in the LFP time-locked to the stimulus onset. To obtain the VEP we first averaged the LFP-response in each of the 16-channels over all available trials, eliminating all components that are not phase-locked to stimulus onset. Then we computed the CSD from the channel-averages. CSD is computed as the second spatial derivative of a particular electrode and its neighbors (Freeman and Nicholson, 1975; Mitzdorf and Singer, 1979; Schroeder et al., 1991).

$\mathrm{CSD}=\frac{\Phi(\mathrm{z}+\mathrm{h})-2 \Phi(\mathrm{z})+\Phi(\mathrm{z}-h)}{h^{2}}$

with $\Phi$ being the potential (VEP), h the interelectrode spacing and $\mathrm{z}$ the depth of the electrode contact under study. This method itself discards the top and bottom channel. To regain the top and bottom channels we applied the method described by Vaknin (Pettersen et al., 2006; Vaknin et al., 1988). The late, "tonic" part of the neuronal response (200-500 ms after stimulus onset) contains components that are not time-locked to the stimulus onset, but are generated by the dynamics of the neuronal network. To extract the locally generated components at a given contact we computed the bipolar LFP (LFPbp) as the difference between the LFP at its neighboring contacts for every trial to minimize common signals (Trongnetrpunya et al., 2015). The LFPbp was then subjected to spectral analysis methods.

\section{Electrode perpendicularity and intersession alignment}

To interpret the laminar specificity of neuronal activity, each of the 16 channels had to be assigned a putative position within the cortical depth for every session (Figure 1). The position in depth varied across sessions due to manual advancement of the probe and the compressions-relaxation dynamics of the cortical tissue during its penetration. For early visual cortex several studies compared the visually evoked activity with the laminar anatomy after histological reconstruction of recording sites (Kraut et al., 1985; Mitzdorf and Singer, 1979; Schroeder et al., 1991). These studies established that an early (40ms after stimulus onset) current sink in the CSD-profile corresponds to the thalamocortical afferent activity reaching layer IVC. This alignment-criterion has been successfully applied in a number of recent studies of primary visual cortex V1 using laminar electrodes ((Maier et al., 2010; Maier et al., 2011; Self et al., 2013; Spaak et al., 2012; van Kerkoerle et al., 2014). To align electrode position across sessions we computed the CSD-profile across all stimulus presentations of each session and visually identified the channel that featured an early current sink, peaking between 
$473 \quad 35-55 \mathrm{~ms}$ after stimulus onset. Figure 1 illustrates the alignment procedure applied to our data.

\section{Data analysis}

475 All data analysis was performed with custom-written code in the Matlab computing environment (The 476 Mathworks, Natick, Mass., USA).

477 The latency of MUAe responses in each layer was determined by analyzing a model of the visually 478 evoked transient from 0 to $200 \mathrm{~ms}$ after stimulus onset. The model was computed by a fitting 479 procedure adapted from Self et al. (2013) which fitted the sum of two Gaussians and a cumulative 480 Gaussian by a non-linear least-squares fitting procedure (Matlab, Curve Fitting Toolbox):

$$
R(t)=\frac{\mathrm{G}_{1} e^{-0.5\left(\frac{t-\mu_{1}}{\sigma_{1}}\right)^{2}}}{\sqrt{2 \pi} \sigma_{1}}+\frac{\mathrm{G}_{2} e^{-0.5\left(\frac{t-\mu_{2}}{\sigma_{2}}\right)^{2}}}{\sqrt{2 \pi} \sigma_{2}}+0.5 G_{3}\left[1+\operatorname{erf}\left(\frac{t-\mu_{3}}{\sqrt{2 \sigma_{3}^{2}}}\right)\right]
$$

$482 \mathrm{R}(\mathrm{t})$ is the response at time $\mathrm{t}$ defined by the parameters of the three Gaussians, (G: amplitude, $\mu$ : mean, $\sigma$ : standard deviation) and erf is the error function. The function was fitted separately to the responses at each electrode contact to each of the 6 stimulus sizes. Before the fitting procedure the MUAe signal was z-scored relative to the spontaneous activity 0 to $300 \mathrm{~ms}$ before stimulus onset. The stimulus size is negatively correlated to the amplitude of visual responses due to surround suppression. Hence, contrary to Self at al. (2013), we used an absolute criterion to determine the response latency as we sought to compare responses to different stimulus sizes. The latency was defined as the earliest time the model exceeded a z-score of 3.

490 For general spectral analysis of the LFP we computed the spectrogram by applying fast Fourier transformation (FFT, $\mathrm{n}=1024$ ) to data sections from a moving window (width=100 $\mathrm{ms}$, step=10ms). Each data section was "hanning-windowed" before FFT was applied.

We computed the LFP phase-triggered average (PTA) to estimate the phase-amplitude-coupling of oscillations between electrode contacts in the gamma frequency band. First, we extracted the phase providing signal ( $\left.S_{\text {phase }}\right)$ from a reference channel by band-pass filtering (butterworth, $n=3,30-55 \mathrm{~Hz}$ ). Then, the broadband signal of the amplitude providing signal $\left(S_{\mathrm{amp}}\right)$ was re-aligned to the troughs of $\mathrm{S}_{\text {phase }}$ and averaged within $+/-25 \mathrm{~ms}$ of each alignment point to yield the PTA-histogram. To quantify the coupling and the phase relationship between $S_{\text {phase }}$ and $S_{\text {amp }}$ we extracted phase and power of the PTA-histograms by means of FFT-analysis. The direction of coupling between the LFPbp of different channels was analysed by means of nonconditional and conditional granger-causality-analysis ( $\left.\mathrm{GC}_{\mathrm{non}}, \mathrm{GC}_{\mathrm{cond}}\right)$. Here, we used the MVGC-

502 toolbox, which is freely available on the web (Barnett and Seth, 2014). For $\mathrm{GC}_{\text {non }}$ as well as for $\mathrm{GC}_{\text {cond }}$ 503 we computed granger-causality indices (GCI) for a stretch of 256 time bins from $200 \mathrm{~ms}$ after stimulus 504 onset on. For the multivariate autoregressive model we used a fixed model order of $50(\sim 50 \mathrm{~ms}$ $505 @ 1017 \mathrm{~Hz}$ ). To extract causal unidirectionality between a certain channel pair we substracted GCIs 
506 calculated in one direction from the GCIs in the reverse direction. For GC $_{\text {non }}$ we computed GCIs for

507 all channel combinations within an experiment. For the $\mathrm{GC}_{\text {cond }}$ we restricted channels pairs to all

508 channel combinations between $+750 \mu \mathrm{m}$ above and $-450 \mu \mathrm{m}$ below the alignment channel since these

509 depth were recorded from in all experiments across the dataset.

510

511 
bioRxiv preprint doi: https://doi.org/10.1101/2020.07.10.197566; this version posted July $10,2020$. The copyright holder for this preprint (which was not certified by peer review) is the author/funder. All rights reserved. No reuse allowed without permission.

512

513

514

515

516

517

518

519

520

521

522

523

524

525

526

527

528

529

530

531

532

533

534

535

536

537

538

539

540

541

542

543

544

545

546

547

548

549

550

551

552

553

554

555

556

557

558

559

560

561

562

563

564

565

566

567

568

569

570

571

572

573

574

\section{References}

Alitto, H.J., and Usrey, W.M. (2008). Origin and dynamics of extraclassical suppression in the lateral geniculate nucleus of the macaque monkey. Neuron 57, 135-146.

Angelucci, A., Levitt, J.B., and Lund, J.S. (2002). Anatomical origins of the classical receptive field and modulatory surround field of single neurons in macaque visual cortical area V1. Prog Brain Res 136, 373388.

Bair, W., Cavanaugh, J.R., and Movshon, J.A. (2003). Time course and time-distance relationships for surround suppression in macaque V1 neurons. J Neurosci 23, 7690-7701.

Bair, W., Cavanaugh, J.R., Smith, M.A., and Movshon, J.A. (2002). The timing of response onset and offset in macaque visual neurons. J Neurosci 22, 3189-3205.

Barnett, L., and Seth, A.K. (2014). The MVGC multivariate Granger causality toolbox: a new approach to Granger-causal inference. J Neurosci Methods 223, 50-68.

Bastos, A.M., Vezoli, J., Bosman, C.A., Schoffelen, J.M., Oostenveld, R., Dowdall, J.R., De Weerd, P., Kennedy, H., and Fries, P. (2015a). Visual areas exert feedforward and feedback influences through distinct frequency channels. Neuron 85, 390-401.

Bastos, A.M., Vezoli, J., and Fries, P. (2015b). Communication through coherence with inter-areal delays. Curr Opin Neurobiol 31, 173-180.

Bauer, R., Brosch, M., and Eckhorn, R. (1995). Different rules of spatial summation from beyond the receptive field for spike rates and oscillation amplitudes in cat visual cortex. Brain Res 669, 291-297.

Bijanzadeh, M., Nurminen, L., Merlin, S., Clark, A.M., and Angelucci, A. (2018). Distinct Laminar Processing of Local and Global Context in Primate Primary Visual Cortex. Neuron.

Bollimunta, A., Mo, J., Schroeder, C.E., and Ding, M. (2011). Neuronal mechanisms and attentional modulation of corticothalamic alpha oscillations. J Neurosci 31, 4935-4943.

Bosman, C.A., Schoffelen, J.M., Brunet, N., Oostenveld, R., Bastos, A.M., Womelsdorf, T., Rubehn, B., Stieglitz, T., De Weerd, P., and Fries, P. (2012). Attentional stimulus selection through selective synchronization between monkey visual areas. Neuron 75, 875-888.

Buffalo, E.A., Fries, P., and Desimone, R. (2004). Layer-specific attentional modulation in early visual areas. Society for Neuroscience, abstracts $35,717.716$.

Buffalo, E.A., Fries, P., Landman, R., Buschman, T.J., and Desimone, R. (2011). Laminar differences in gamma and alpha coherence in the ventral stream. Proc Natl Acad Sci U S A 108, 11262-11267.

Buschman, T.J., and Miller, E.K. (2007). Top-down versus bottom-up control of attention in the prefrontal and posterior parietal cortices. Science 315, 1860-1862.

Buschman, T.J., and Miller, E.K. (2009). Serial, covert shifts of attention during visual search are reflected by the frontal eye fields and correlated with population oscillations. Neuron 63, 386-396.

Callaway, E.M. (1998). Local circuits in primary visual cortex in the macaque monkey. Annual Review of Neuroscience 21, 47-74.

Callaway, E.M. (2004). Feedforward, feedback and inhibitory connections in primate visual cortex. Neural Netw $17,625-632$.

Dougherty, K., Cox, M.A., Ninomiya, T., Leopold, D.A., and Maier, A. (2017). Ongoing Alpha Activity in V1 Regulates Visually Driven Spiking Responses. Cereb Cortex 27, 1113-1124.

Douglas, R.J., and Martin, K.A. (2004). Neuronal circuits of the neocortex. Annu Rev Neurosci 27, 419-451.

Douglas, R.J., Martin, K.A.C., and Whitteridge, D. (1989). A canonical microcircuit for neocortex. Neural Computation 1, 480-488.

Einevoll, G.T., Kayser, C., Logothetis, N.K., and Panzeri, S. (2013). Modelling and analysis of local field potentials for studying the function of cortical circuits. Nat Rev Neurosci 14, 770-785.

Freeman, J.A., and Nicholson, C. (1975). Experimental optimization of current source-density technique for anuran cerebellum. J Neurophysiol 38, 369-382.

Fries, P. (2015). Rhythms for Cognition: Communication through Coherence. Neuron 88, 220-235.

Gieselmann, M.A., and Thiele, A. (2008). Comparison of spatial integration and surround suppression characteristics in spiking activity and the local field potential in macaque V1. Eur J Neurosci 28, 447-459.

Gray, H., Bertrand, H., Mindus, C., Flecknell, P., Rowe, C., and Thiele, A. (2016). Physiological, Behavioral, and Scientific Impact of Different Fluid Control Protocols in the Rhesus Macaque (Macaca mulatta). eNeuro 3.

Haegens, S., Barczak, A., Musacchia, G., Lipton, M.L., Mehta, A.D., Lakatos, P., and Schroeder, C.E. (2015). Laminar Profile and Physiology of the alpha Rhythm in Primary Visual, Auditory, and Somatosensory Regions of Neocortex. J Neurosci 35, 14341-14352.

Haufe, S., Nikulin, V.V., Muller, K.R., and Nolte, G. (2013). A critical assessment of connectivity measures for EEG data: a simulation study. Neuroimage 64, 120-133.

Hupé, J.M., James, A.C., Payne, B.R., Lomber, S.G., Girard, P., and Bullier, J. (1998). Cortical feedback improves discrimination between figure and background by V1, V2 and V3 neurons. Nature 394, 784-797.

Jia, X., Smith, M.A., and Kohn, A. (2011). Stimulus selectivity and spatial coherence of gamma components of the local field potential. J Neurosci 31, 9390-9403. 
bioRxiv preprint doi: https://doi.org/10.1101/2020.07.10.197566; this version posted July $10,2020$. The copyright holder for this preprint (which was not certified by peer review) is the author/funder. All rights reserved. No reuse allowed without permission.

Kraut, M.A., Arezzo, J.C., and Vaughan, H.G., Jr. (1985). Intracortical generators of the flash VEP in monkeys. Electroencephalogr Clin Neurophysiol 62, 300-312.

Lund, J.S. (1988). Anatomical organization of macaque monkey striate visual cortex. Annu Rev Neurosci 11, 253-288.

Maier, A., Adams, G.K., Aura, C., and Leopold, D.A. (2010). Distinct superficial and deep laminar domains of activity in the visual cortex during rest and stimulation. Front Syst Neurosci 4.

Maier, A., Aura, C.J., and Leopold, D.A. (2011). Infragranular sources of sustained local field potential responses in macaque primary visual cortex. J Neurosci 31, 1971-1980.

Markov, N.T., Ercsey-Ravasz, M., Van Essen, D.C., Knoblauch, K., Toroczkai, Z., and Kennedy, H. (2013). Cortical high-density counterstream architectures. Science 342, 1238406.

Markov, N.T., Ercsey-Ravasz, M.M., Ribeiro Gomes, A.R., Lamy, C., Magrou, L., Vezoli, J., Misery, P., Falchier, A., Quilodran, R., Gariel, M.A., et al. (2014). A weighted and directed interareal connectivity matrix for macaque cerebral cortex. Cereb Cortex 24, 17-36.

Mitzdorf, U. (1987). Properties of the evoked potential generators: current source-density analysis of visually evoked potentials in the cat cortex. Int J Neurosci 33, 33-59.

Mitzdorf, U., and Singer, W. (1979). Excitatory synaptic ensemble properties in the visual cortex of the macaque monkey: a current source density analysis of electrically evoked potentials. J Comp Neurol 187, 71-83.

Nassi, J.J., Lomber, S.G., and Born, R.T. (2013). Corticocortical feedback contributes to surround suppression in V1 of the alert primate. J Neurosci 33, 8504-8517.

Nowak, L.G., Munk, M.H.J., Girard, P., and Bullier, J. (1995). Visual latencies in areas V1 and V2 of the macaque monkey. Visual Neurosci 12, 371-384.

Nurminen, L., Merlin, S., Bijanzadeh, M., Federer, F., and Angelucci, A. (2018). Top-down feedback controls spatial summation and response amplitude in primate visual cortex. Nat Commun 9, 2281.

Pettersen, K.H., Devor, A., Ulbert, I., Dale, A.M., and Einevoll, G.T. (2006). Current-source density estimation based on inversion of electrostatic forward solution: effects of finite extent of neuronal activity and conductivity discontinuities. J Neurosci Methods 154, 116-133.

Ray, S., and Maunsell, J.H. (2010). Differences in gamma frequencies across visual cortex restrict their possible use in computation. Neuron 67, 885-896.

Roberts, M.J., Lowet, E., Brunet, N.M., Ter Wal, M., Tiesinga, P., Fries, P., and De Weerd, P. (2013). Robust gamma coherence between macaque $V 1$ and $V 2$ by dynamic frequency matching. Neuron 78, 523-536.

Rockland, K.S., and Pandya, D.N. (1979). Laminar origins and terminations of cortical connections of the occipital lobe in the rhesus monkey. Brain Res 179, 3-20.

Sceniak, M.P., Hawken, M.J., and Shapley, R. (2001). Visual spatial characterization of macaque V1 neurons. J Neurophysiol 85, 1873-1887.

Schroeder, C.E., Tenke, C.E., Givre, S.J., Arezzo, J.C., and Vaughan, H.G., Jr. (1991). Striate cortical contribution to the surface-recorded pattern-reversal VEP in the alert monkey. Vision Res 31, 1143-1157.

Self, M.W., van Kerkoerle, T., Super, H., and Roelfsema, P.R. (2013). Distinct roles of the cortical layers of area $\mathrm{V} 1$ in figure-ground segregation. Curr Biol 23, 2121-2129.

Spaak, E., Bonnefond, M., Maier, A., Leopold, D.A., and Jensen, O. (2012). Layer-specific entrainment of gamma-band neural activity by the alpha rhythm in monkey visual cortex. Curr Biol 22, 2313-2318.

Super, H., and Roelfsema, P.R. (2005). Chronic multiunit recordings in behaving animals: advantages and limitations. Prog Brain Res 147, 263-282.

Thiele, A., Delicato, L.S., Roberts, M.J., and Gieselmann, M.A. (2006). A novel electrode-pipette design for simultaneous recording of extracellular spikes and iontophoretic drug application in awake behaving monkeys. J Neurosci Methods 158, 207-211.

Tiesinga, P., and Sejnowski, T.J. (2009). Cortical enlightenment: are attentional gamma oscillations driven by ING or PING? Neuron 63, 727-732.

Trongnetrpunya, A., Nandi, B., Kang, D., Kocsis, B., Schroeder, C.E., and Ding, M. (2015). Assessing Granger Causality in Electrophysiological Data: Removing the Adverse Effects of Common Signals via Bipolar Derivations. Front Syst Neurosci 9, 189.

Vaknin, G., DiScenna, P.G., and Teyler, T.J. (1988). A method for calculating current source density (CSD) analysis without resorting to recording sites outside the sampling volume. J Neurosci Methods 24, 131135.

van Kerkoerle, T., Self, M.W., Dagnino, B., Gariel-Mathis, M.A., Poort, J., van der Togt, C., and Roelfsema, P.R. (2014). Alpha and gamma oscillations characterize feedback and feedforward processing in monkey visual cortex. Proc Natl Acad Sci U S A 111, 14332-14341.

Vinck, M., Huurdeman, L., Bosman, C.A., Fries, P., Battaglia, F.P., Pennartz, C.M., and Tiesinga, P.H. (2015). How to detect the Granger-causal flow direction in the presence of additive noise? Neuroimage 108,301 318.

Xing, D., Yeh, C.I., Burns, S., and Shapley, R.M. (2012). Laminar analysis of visually evoked activity in the primary visual cortex. Proc Natl Acad Sci U S A 109, 13871-13876. 


\section{Figures}

639

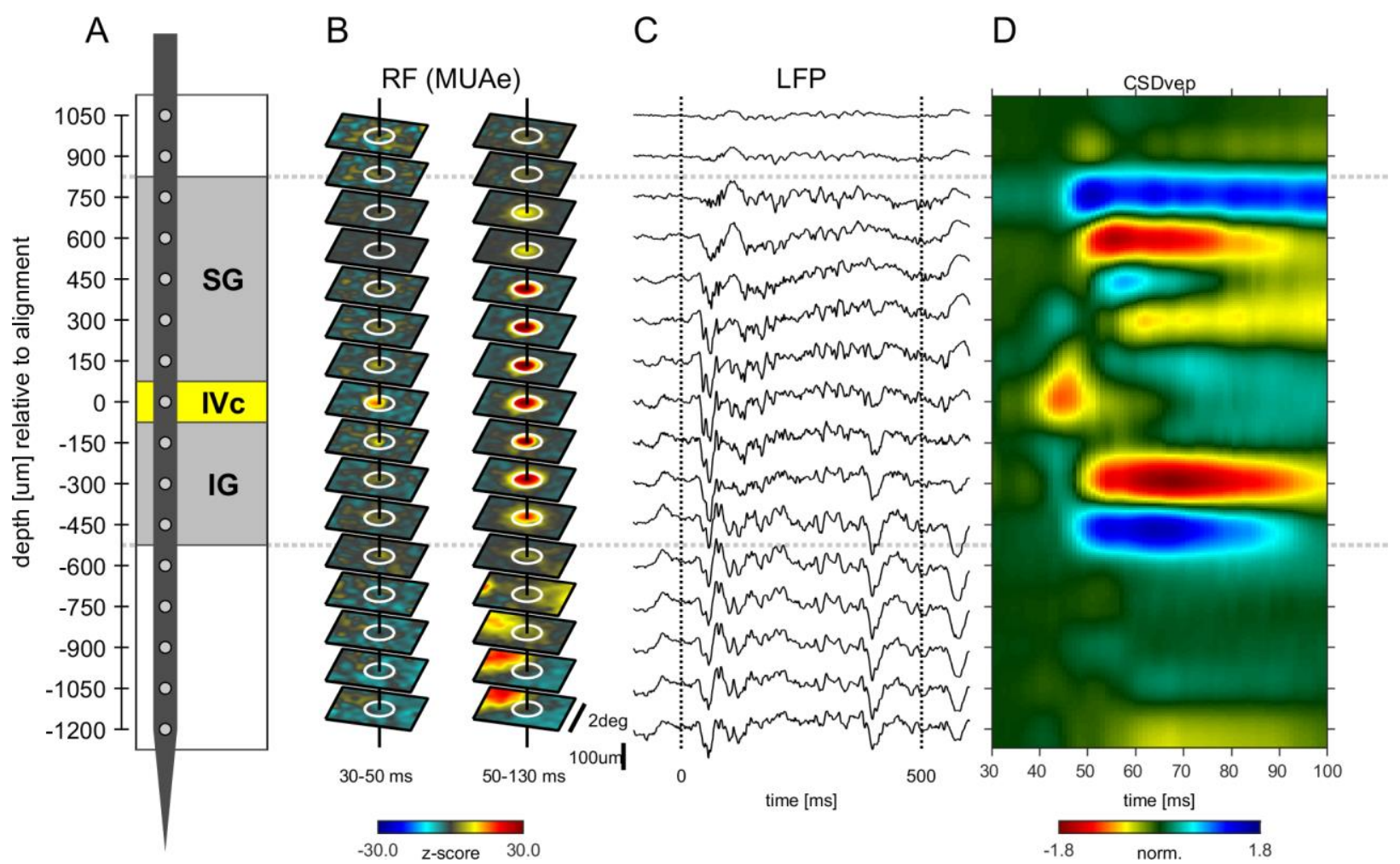

Figure 1: Example experiment A) Illustration of probe position and electrode contacts in relation to cortical gray matter during a recording session. Position in depth is aligned to input layer IVC at $0 \mu \mathrm{m}$. Gray dotted lines show boundaries of neuronal responsivity across panels (A-E). B) Minimum response fields of MUAe for each channel. Vertical solid black line shows RF center, white circle indicates a $1^{\circ}$ diameter. Left column shows low latency response between 30 and 50 ms after stimulus onset. Right column shows response between 50 and 130 ms after stimulus onset. Activity is z-scored to baseline period. C) Response profile of LFP activity for a single trial with a stimulus of $3^{\circ}$ in diameter. D) Depth profile of the CSD signal averaged across all stimulus presentations. Red colors indicate current-sinks and blue colors indicate current-sources. 
A
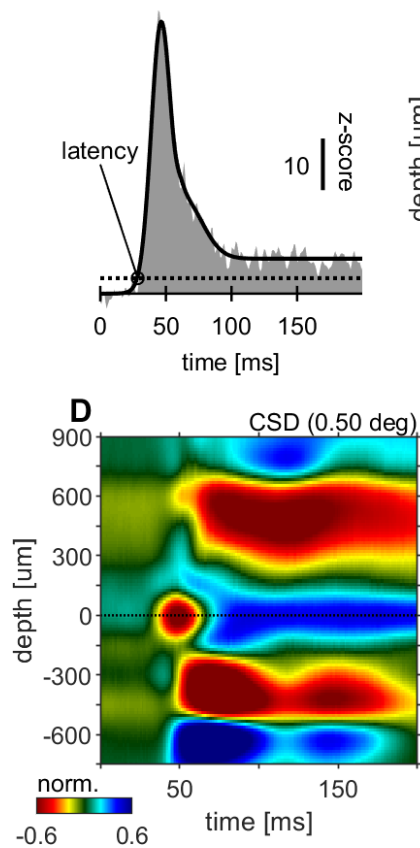
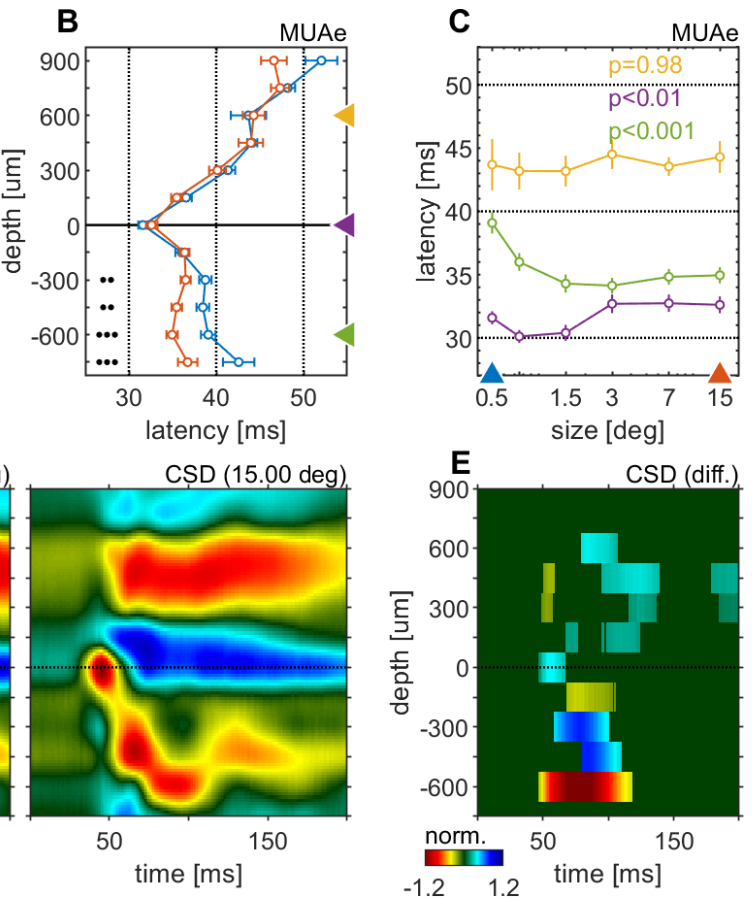

Figure 2: Centre surround effects on early responses of MUAe and CSD depth-profiles. (A) Initial MUAe response used to derive response latencies across depths. The example is from the alignment channel of the experiment shown in Figure 1. (B) Depth-profiles of MUAe latency. Black dots indicate significant levels of latency differences (signed-rank test, 3:p<0.0001, 2:p $<0.001,1: p<0.01$; FDR-corrected) between large (red, $15^{\circ}$ ) and small (blue, $0.5^{\circ}$ ) stimulus. (C) Sizetuning of MUAe latency for three laminar compartments (yellow: supragranular, purple: granular, green: infragranular). Latencies from supragranular and in infragranular layers were averaged across two adjacent channels (see triangles on depth in B). P-Values indicate size dependent differences in latency for the 3 contacts (ANOVA. (D) CSD depth-profile for a small (left, $0.5^{\circ}$ ) and a large (right, $15^{\circ}$ ) stimulus, averaged across experiments. Profile of each session was normalized to the amplitude of the early current sink in putative layer IVc (pos. peak at $0 \mu \mathrm{m}$ between $40-70 \mathrm{~ms}$ ). (E) Depth-profile showing significant differences between the two depth-profiles in $\mathrm{D}(\mathrm{p}<0.05$, cluster-mass-test). 

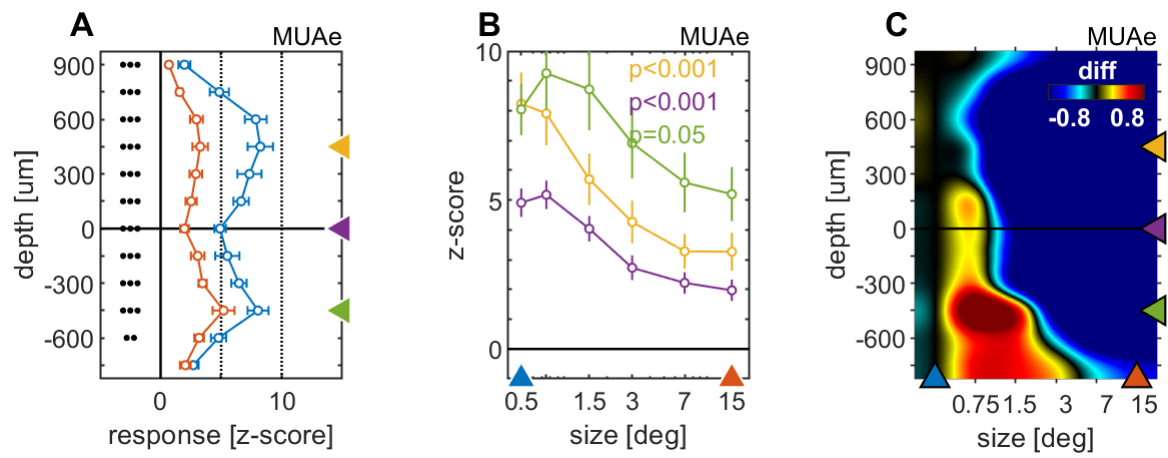

Figure 3: Centre surround effects on late/sustained (200-500 ms) response properties of MUAe. (A) Depth-profiles show average z-scores (normalized to spontaneous activity) across experiments and errorbars represent the s.e.m. for two selected stimulus sizes. Grouped black dots indicate significant levels of signed-rank test (3:p<0.0001, 2:p<0.001, 1:p $<0.01$; FDR-corrected) between small $\left(0.5^{\circ}\right.$, blue $)$ and large $\left(15^{\circ}\right.$, red) stimulus diameter (B) Size-tuning and responsedepth-profiles of MUAe data for all grating diameters and three laminar locations. Yellow, green and purple lines/symbols refer to different depths in the supra-granular $(+450 \mu \mathrm{m})$, infra-granular $(-450 \mu \mathrm{m})$ and granular (alignment, $0 \mu \mathrm{m}$ ) regions. (C) Color-map representation of the differences of MUAe response relative to reference size $0.5^{\circ}$. 
A
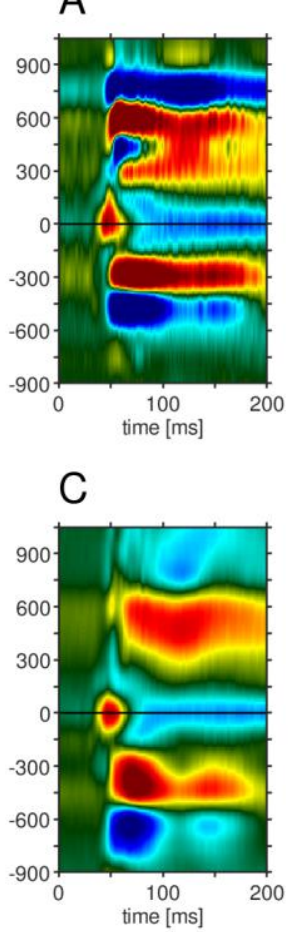

B
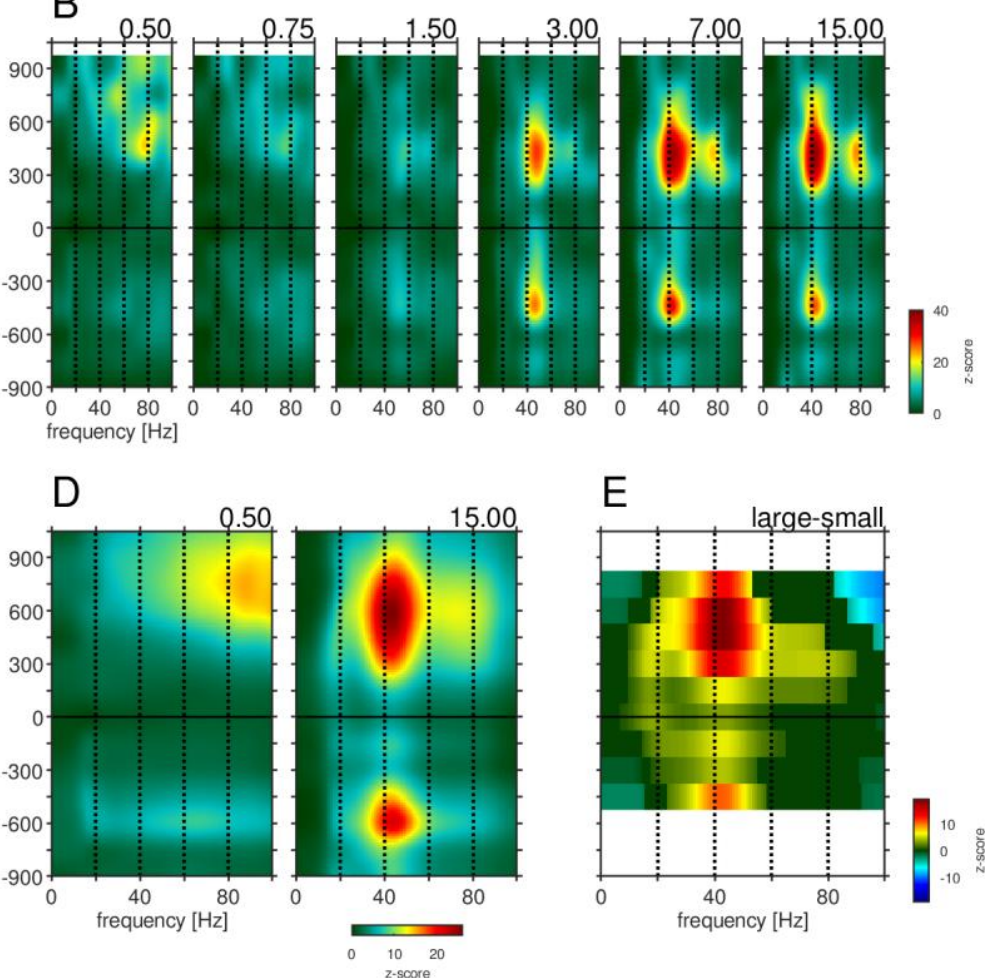

E

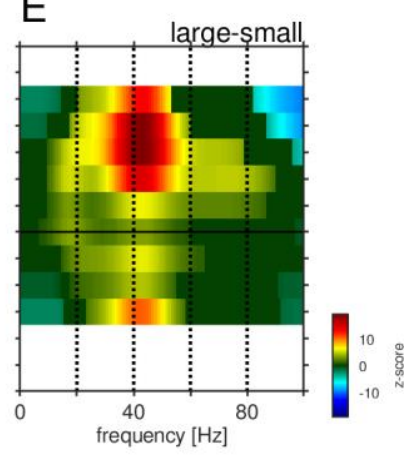

Figure 4: Depth-profile of spectral density for "small" and "large" stimuli. A) Depth profile of the CSD signal for a $0.5^{\circ}$ grating in the example experiment (see Figure 1). Horizontal solid white lines indicate cortical lamination from anatomical data. B) Depth-profile of LFPbp-spectral-density (normalized to spontaneous activity as z-score) for all six grating diameter in the example experiment. C) Depth profile of the CSD signal for a $0.5^{\circ}$ grating across all experiments $(\mathrm{n}=24)$. D) Depth-profile of LFPbp-spectral-density for "small" $\left(0.5^{\circ}\right)$ and "large" $\left(7.0^{\circ}\right)$ gratings across all experiments. E) Depth-profile of the difference in spectral-density between "large" and "small" gratings. Warm and cold colors indicate increases and decreases with stimulus size. White color indicates cortical depths which were not covered by all of the $\mathrm{n}=24$ experiments. Dark green color indicate non-significant differences according to a "non-parametric cluster analysis" or "cluster-mass-test" (Bullmore et al., 1999; Maris and Oostenveld, 2007; Self et al., 2013). 

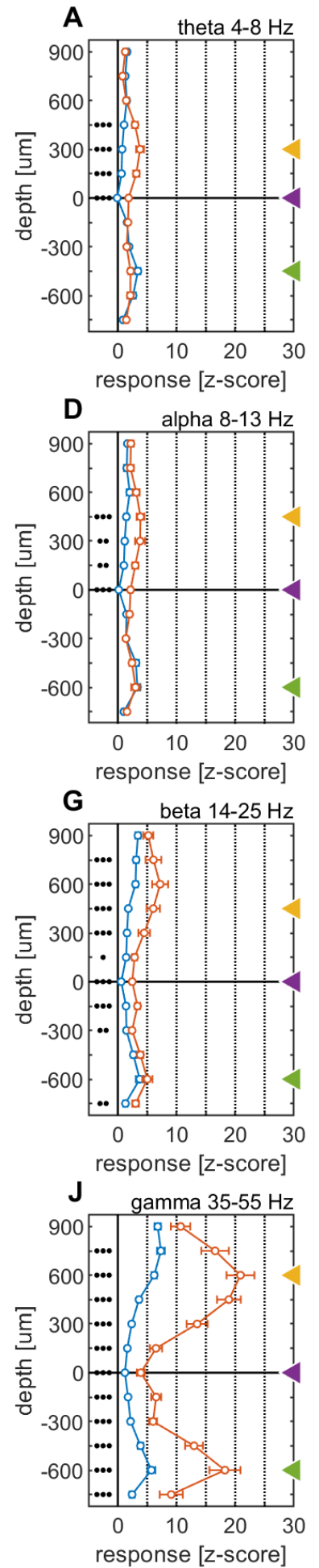
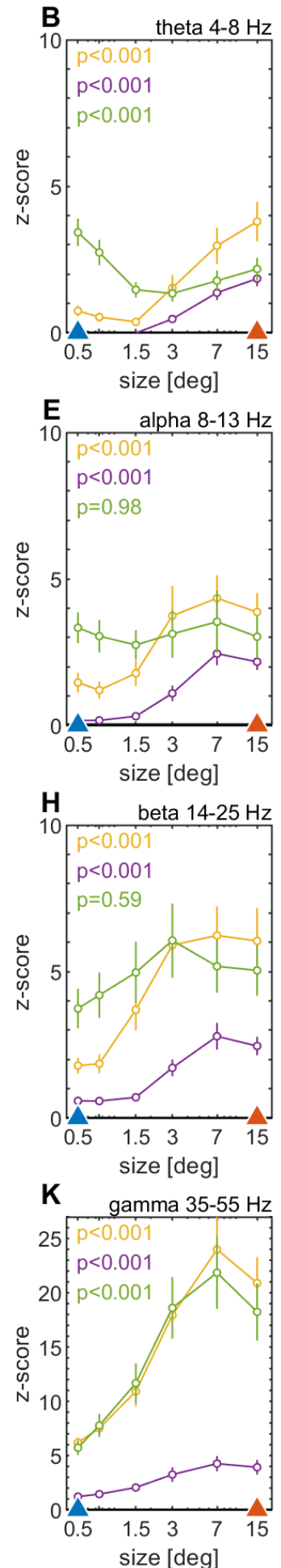
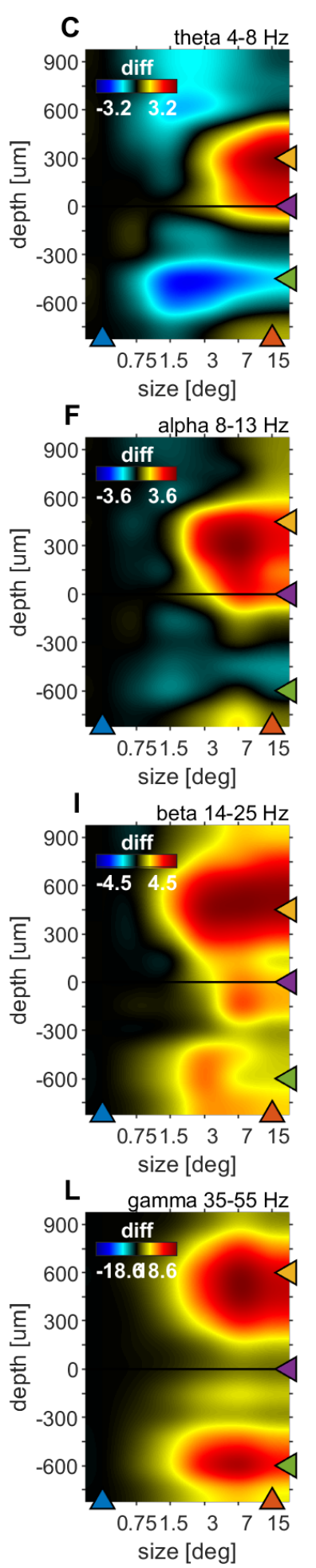

Figure 5 : Centre surround effects on late/sustained (200-500 ms) responses in 4 "classic" frequency bands. A) Size-tuning and response-depth-profiles of average power of LFPbp signal in four spectral bands (theta: 4-8 Hz, alpha: 8-13 Hz, beta: 14-25 Hz, gamma: $35-55 \mathrm{~Hz})$ for small $\left(0.5^{\circ}\right.$, blue $)$ and large $\left(15^{\circ}\right.$, red) stimuli. Grouped black dots indicate significant levels of signed-rank test (3:p<0.0001, 2:p<0.001, 1:p<0.01; FDR-corrected). (B) Size-tuning and response-depth-profiles of LFPbp spectra for all grating diameters and three laminar locations. Yellow, green and purple lines/symbols refer to depths in the supra-granular $(+450 \mu \mathrm{m})$, infra-granular $(-450 \mu \mathrm{m})$ and granular (alignment, $0 \mu \mathrm{m})$ regions. C) Color-map representation of the differences of LFPbp spectral response relative to reference size $0.5^{\circ}$. 


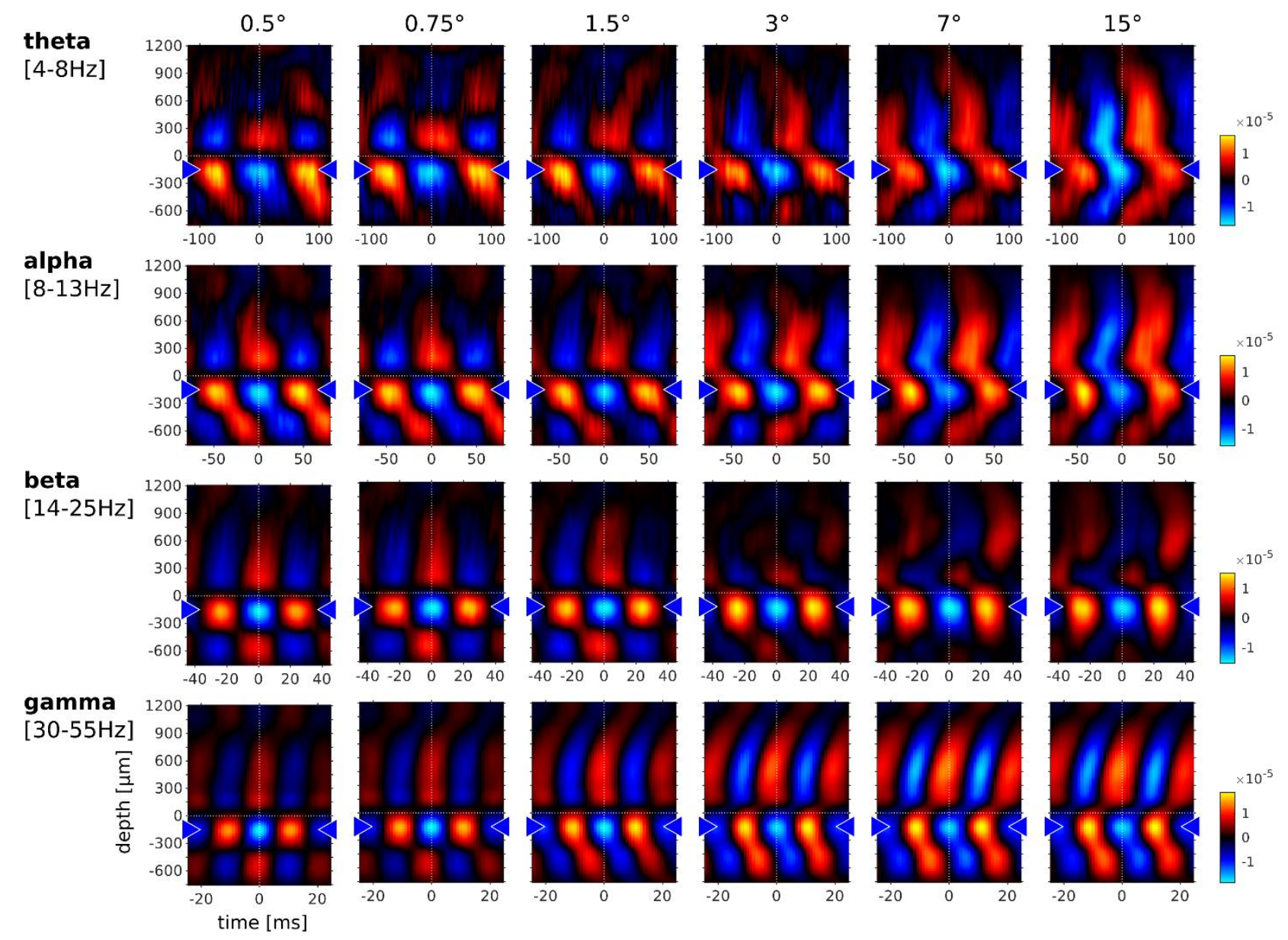

Figure 5: Phase-triggered-average (PTA) of phase-amplitude-coupling between cortical layers in 4 frequency bands. Laminar profiles of cortical oscillations of the LFPbp triggered by the phase of the LFPbp at the reference contact at -150 $\mu \mathrm{m}$ (indicated by the blue triangle). Top row: trigger LFP theta band filtered [4-8 Hz]. Second row: trigger LFP alpha band filtered [8-13 Hz]. Third row: trigger LFP beta band filtered [14-25 Hz]. Bottom row: trigger LFP gamma band filtered [35-55 Hz]). Each plot shows the phase-amplitude-coupling between the reference contact and all other contacts $(-750$ to $1200 \mu \mathrm{m})$ for the sustained response $200-500 \mathrm{~ms}$ after stimulus onset. The color scaling (amplitude of the averaged LFPbp) is the same for all plots within a row. Blue color indicate a trough, red colors indicate a peak in the broadband LFPbp. 

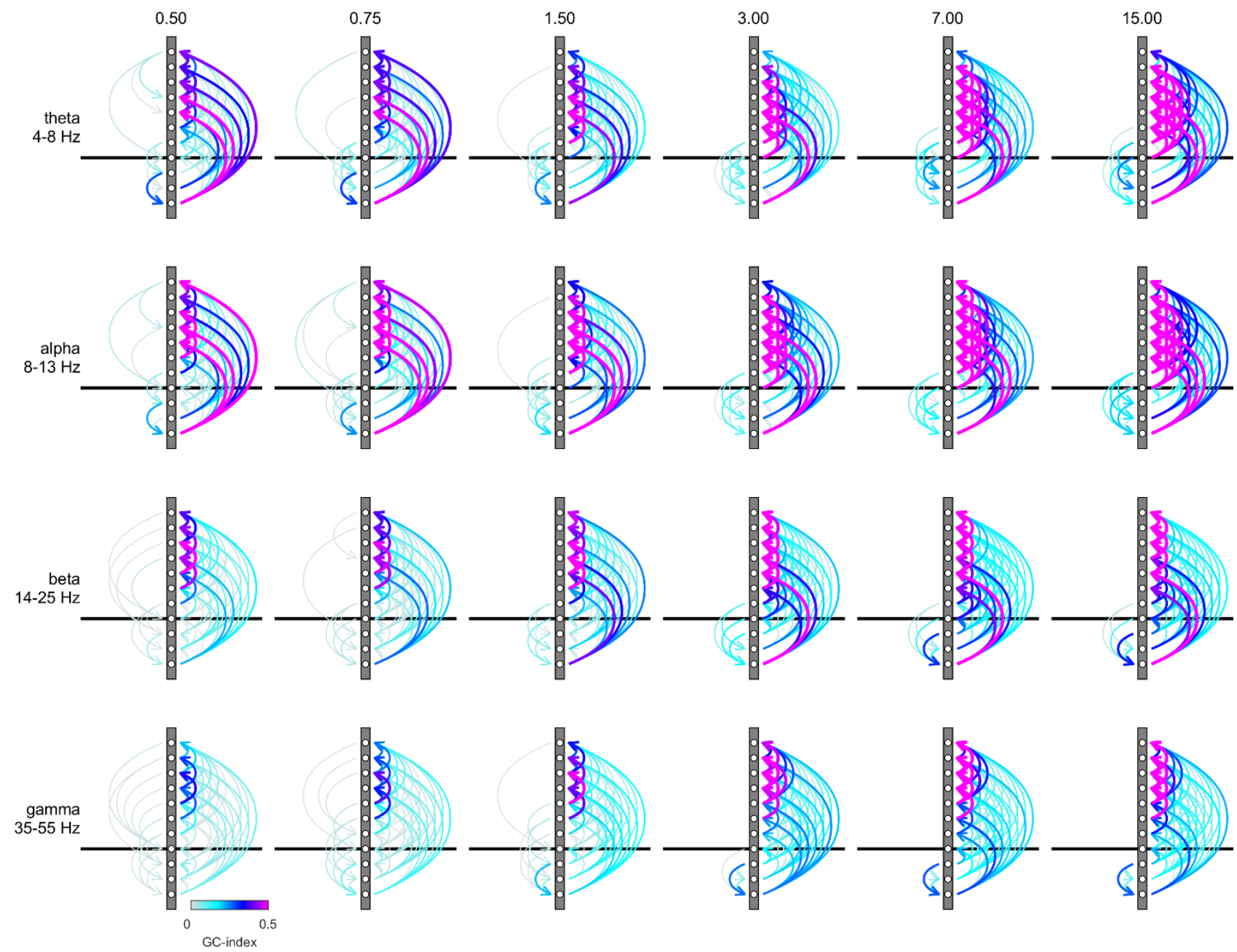

Figure 6: Granger-Causality (GC) analysis reveals directed coupling between cortical layers. Causal-coupling pattern derived from non-conditional GC analysis in the frequency domain and tested with time-reversed GC analysis. GC-indices were integrated across the four classic frequency bands (top row: theta [4-8 Hz], second row: alpha [8-13 Hz], third row: beta [14-25 Hz], bottom row: gamma [35-55 Hz]). Each arrow indicates the dominant direction of causality between a given pair of contacts. The color represents the value of the difference between the GC-indices in each direction. Downward directions are plotted on the left, upward directions are plotted on the right of each profile 


\section{Supplementary materials:}

\section{Stimulus dependence of directed information exchange between cortical layers in macaque $\mathrm{V} 1$}

647 Marc Alwin Gieselmann ${ }^{1}$, Alexander Thiele ${ }^{1 *}$

$648{ }^{1}$ Biosciences Institute, Newcastle University, UK

649

$650 *$ Corresponding and senior author: Alexander Thiele, Biosciences Institute, Newcastle University, 651 Newcastle upon Tyne, NE2 4HH. E-mail: alex.thiele@ncl.ac.uk, Orcid ID: https://orcid.org/0000-0003$652 \underline{4894-0213}$ 
654 Supplementary Figures

A theta $4-8 \mathrm{~Hz}$
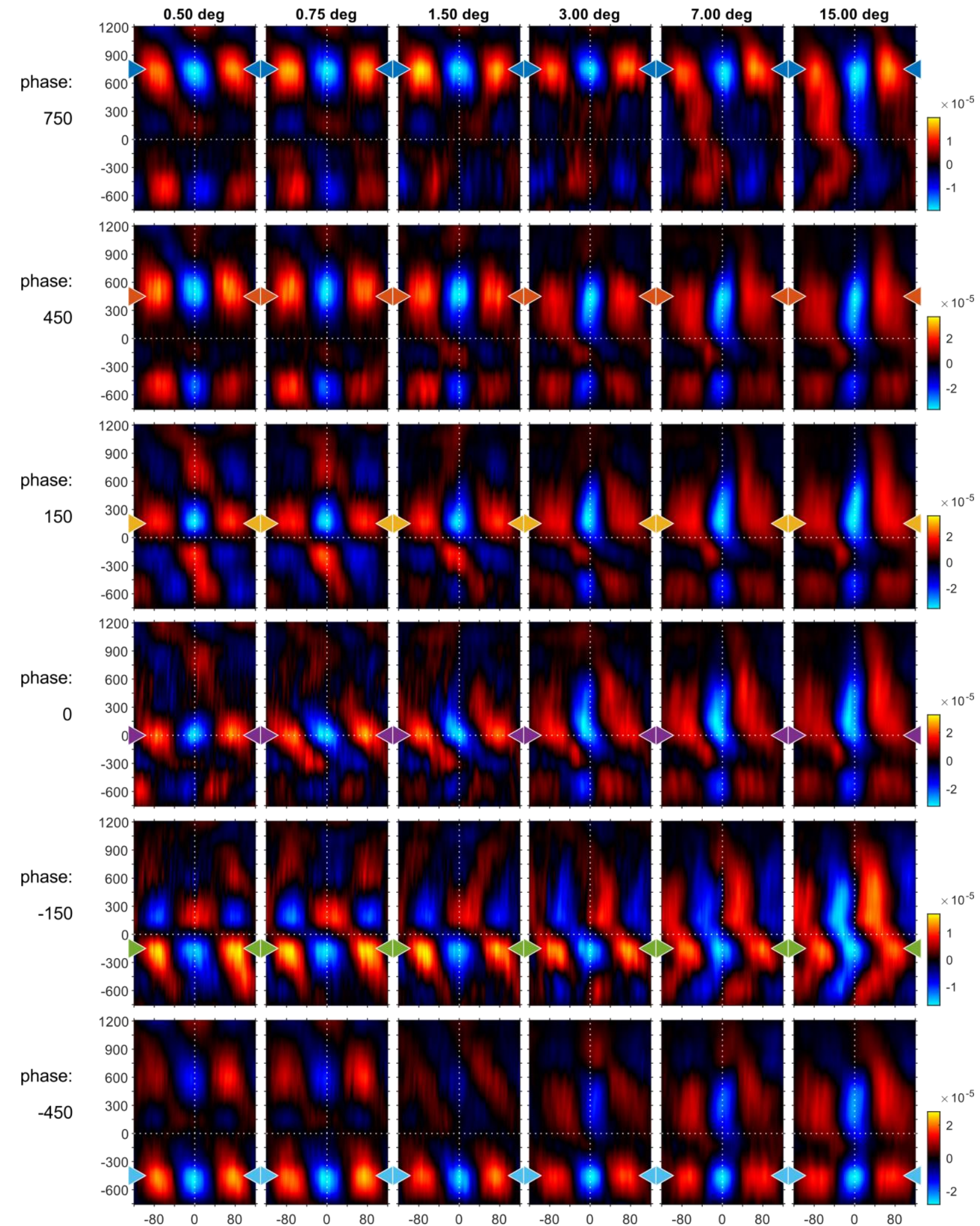

656 Figure S1: Phase-triggered-averages (PTA) of local LFPbp aligned to 6 different reference channels

657 (top to bottom: $750,450,150,-150,-450 \mu \mathrm{m}$ ) for the theta frequency band [4-8 Hz], The figure

658 follows the conventions set out by figure 6 in the main text. 
B

alpha $8-13 \mathrm{~Hz}$
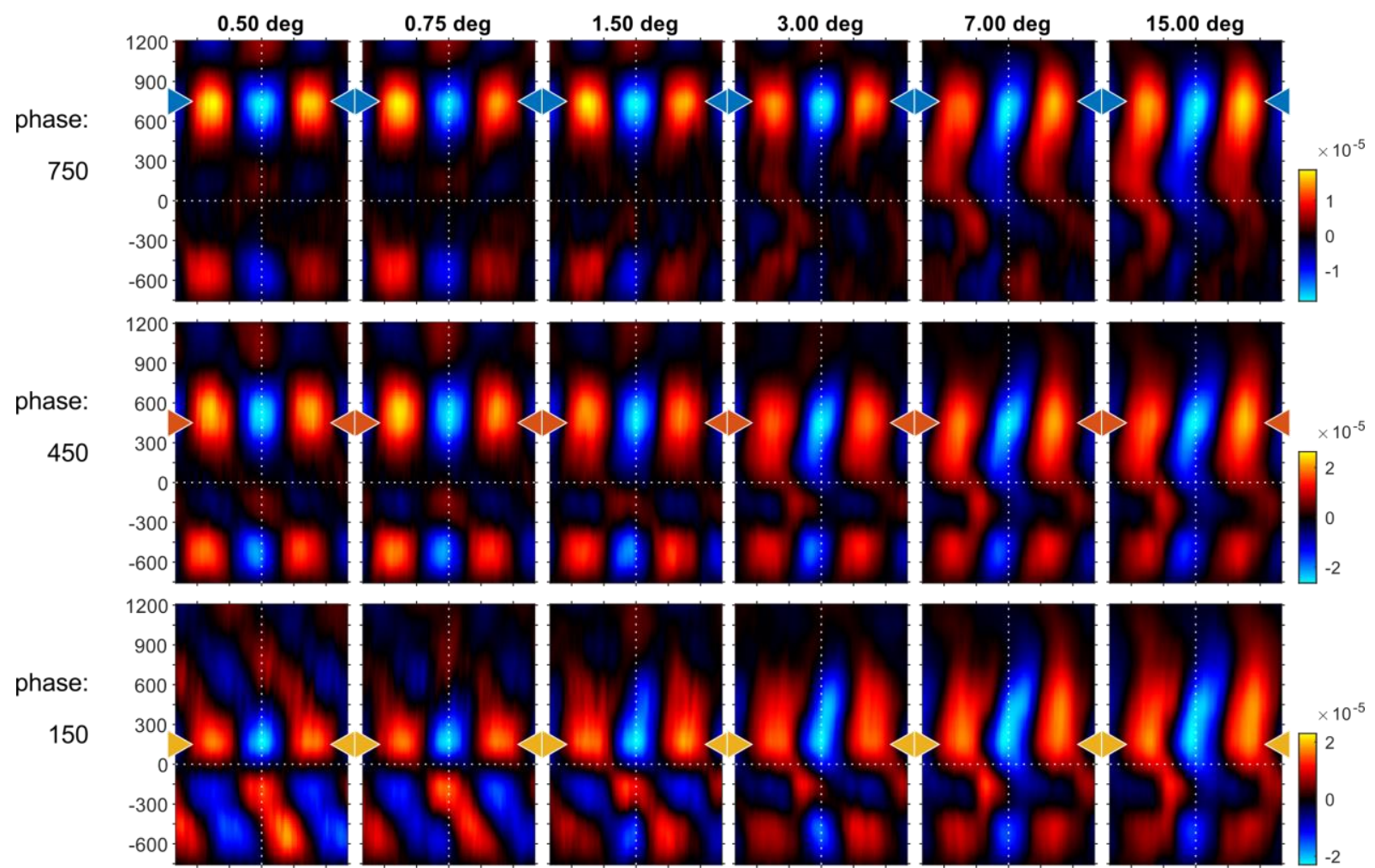

phase:
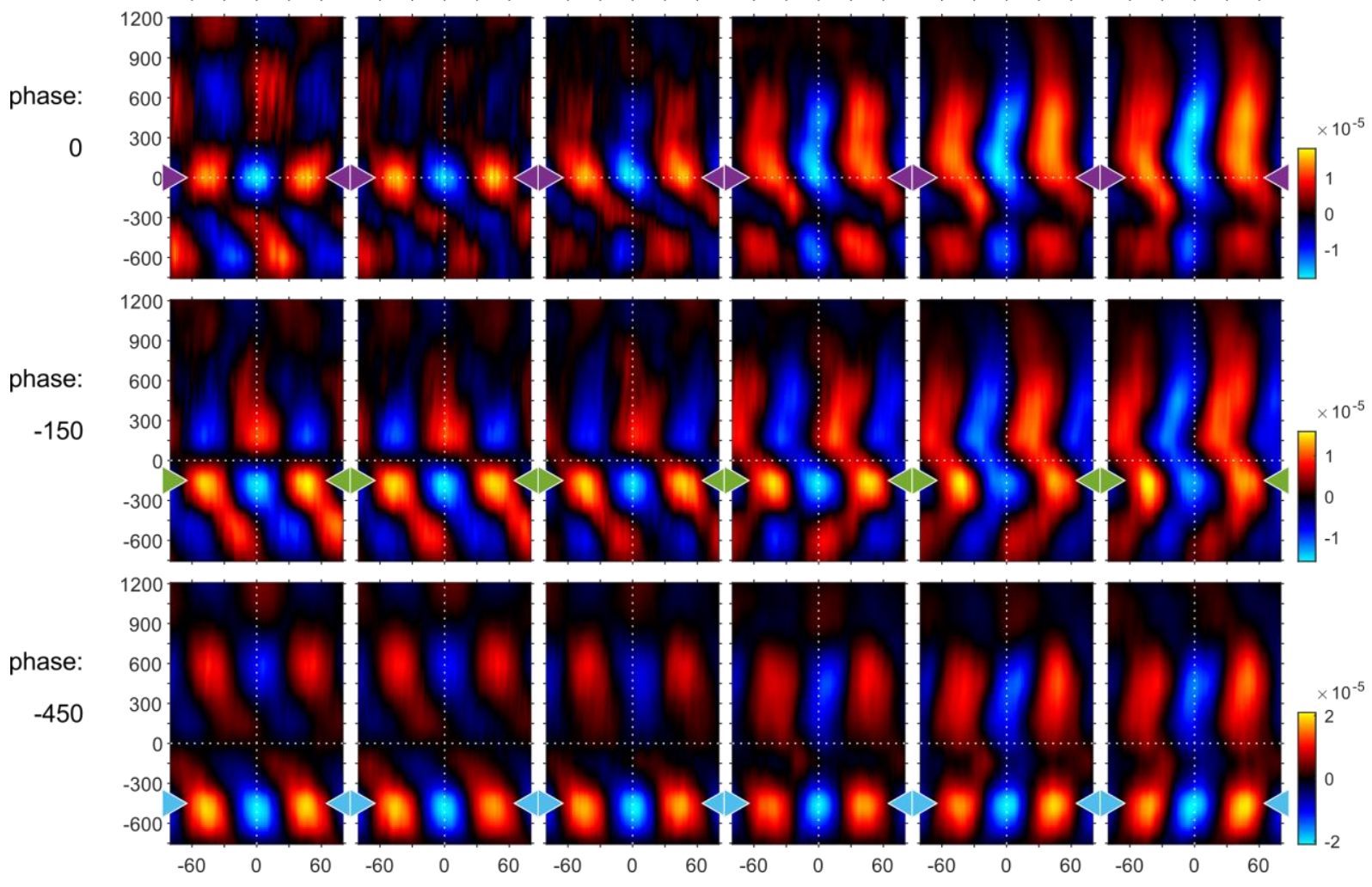

660 Figure S2: Phase-triggered-averages (PTA) of local LFPbp aligned to 6 different reference channels

661 (top to bottom: $750,450,150,-150,-450 \mu \mathrm{m}$ ) for the alpha frequency band [8-13 Hz], The figure

662 follows the conventions set out by figure 6 in the main text. 
C beta $14-25 \mathrm{~Hz}$
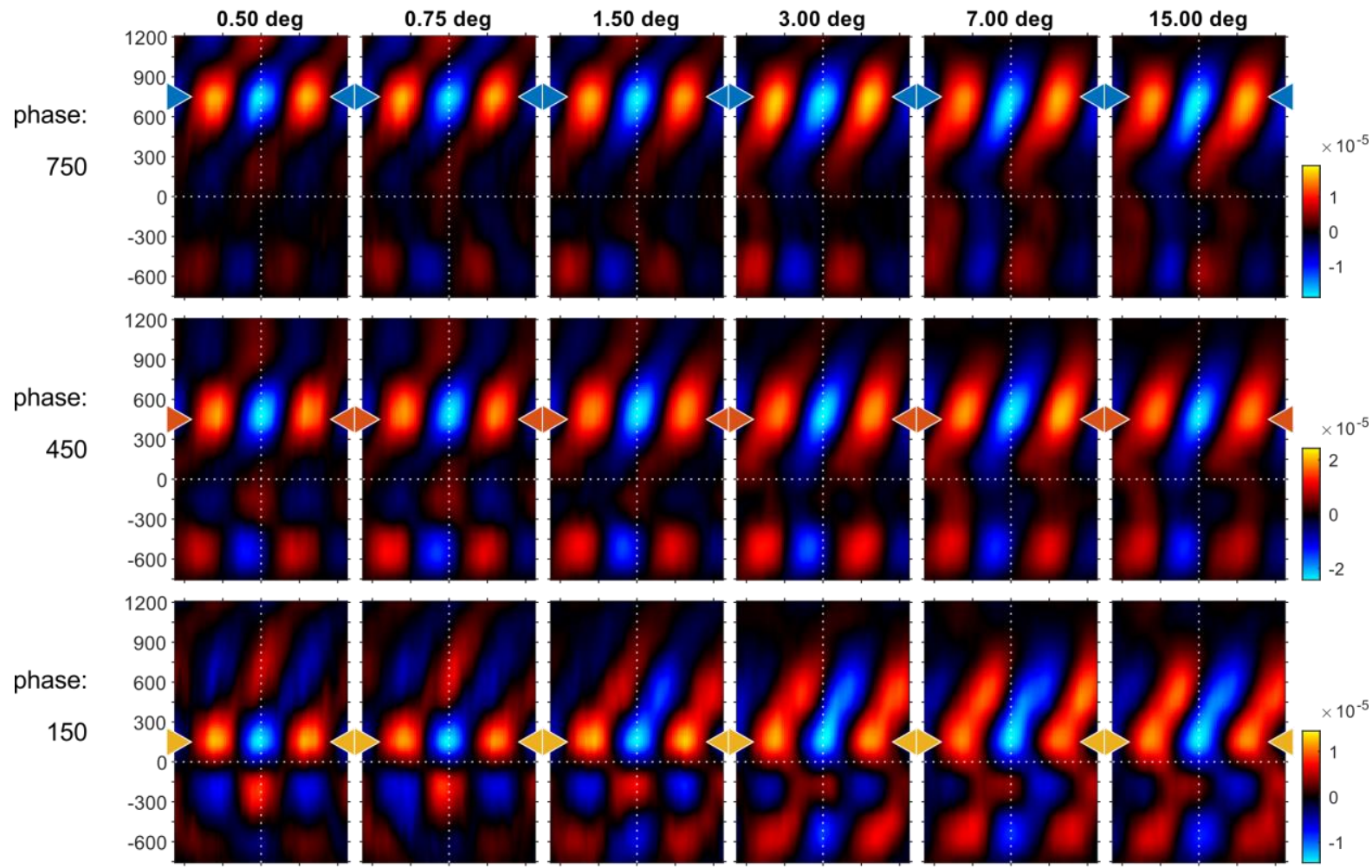

phase:
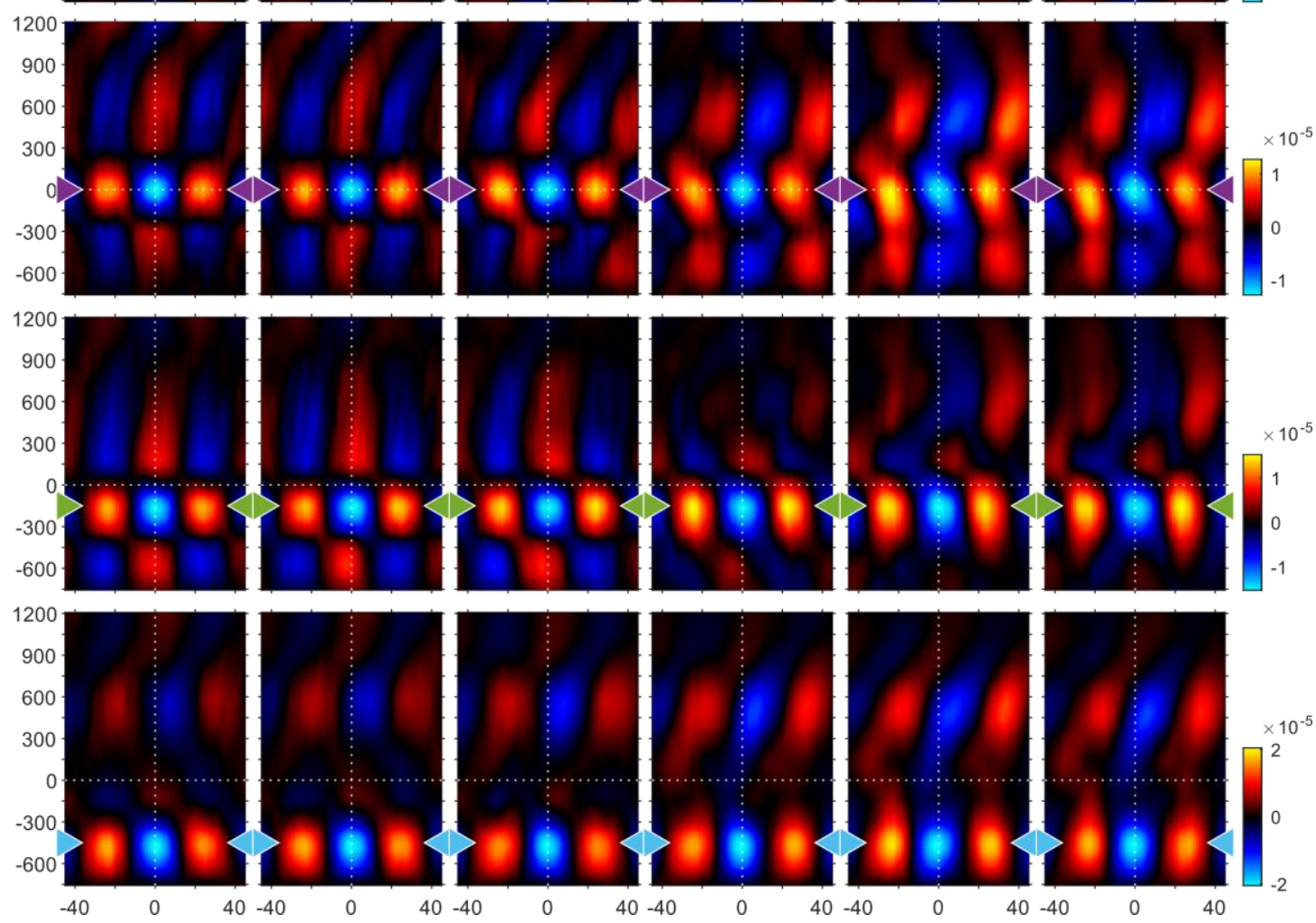

phase:

665 Figure S3: Phase-triggered-averages (PTA) of local LFPbp aligned to 6 different reference channels

666 (top to bottom: $750,450,150,-150,-450 \mu \mathrm{m}$ ) for the beta frequency band [14-25 Hz], The figure

667 follows the conventions set out by figure 6 in the main text. 
D gamma $30-55 \mathrm{~Hz}$
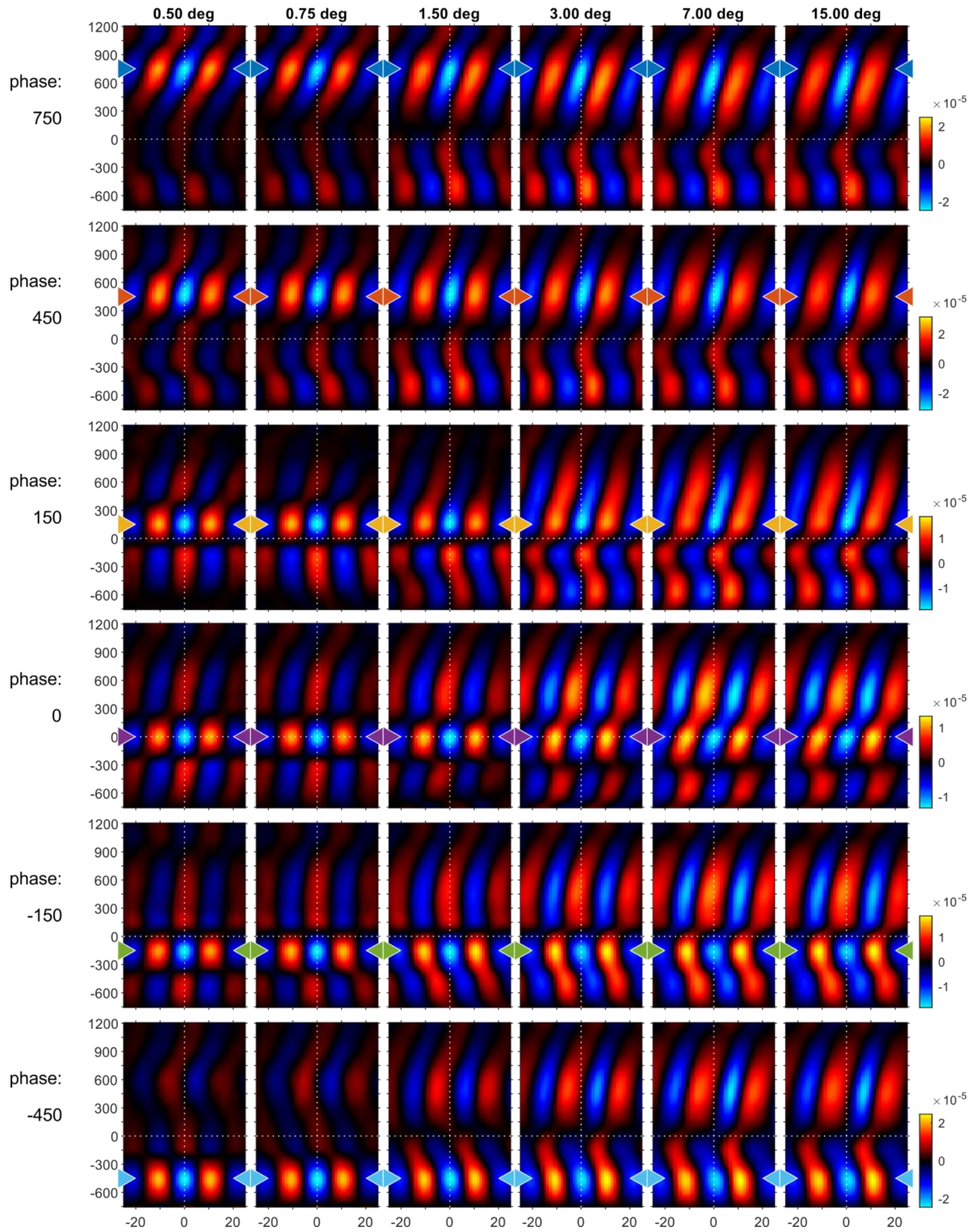

670 Figure S4: Phase-triggered-averages (PTA) of local LFPbp aligned to 6 different reference channels

671 (top to bottom: $750,450,150,-150,-450 \mu \mathrm{m}$ ) for the gamma frequency band [35-55 Hz], The

672 figure follows the conventions set out by figure 6 in the main text. 

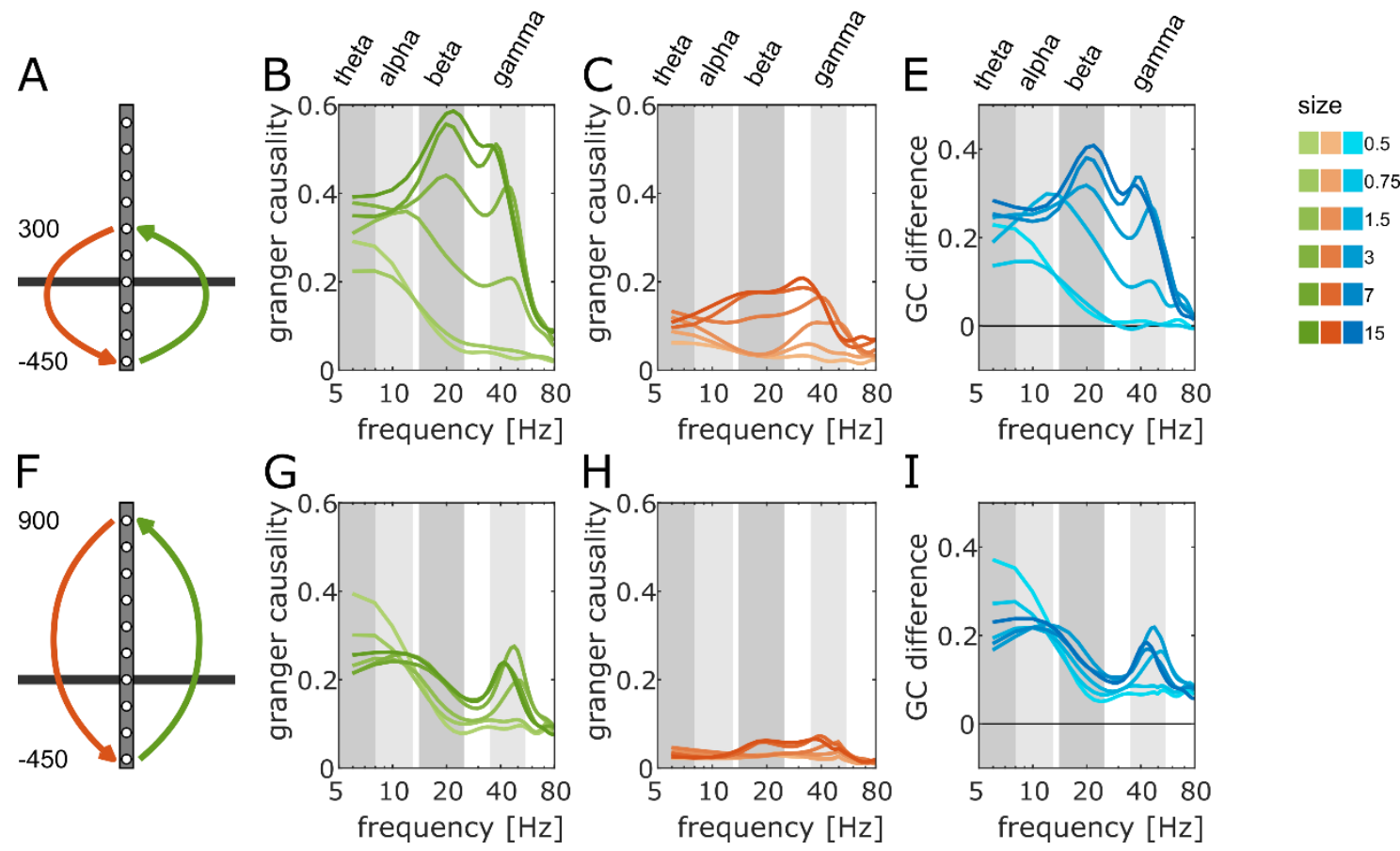

676 Figure S5: Spectral Granger-causality for selected connections between infra-granular and supra-

677 granular layers. (A,F) Diagram of the connections analyzed in panel B-E (-450 to $300 \mu \mathrm{m}$ ) and in

678 panel G-I (-450 to $900 \mu \mathrm{m})$. (B,G) Spectral Granger-causality for the connections in upwards (infra-

679 to supra-granular, green) direction. $(\mathrm{C}, \mathrm{H})$ Spectral Granger-causality for the connections in

680 downwards (infra- to supra-granular, red) direction. (E, I) Net Granger-causality as the difference

681 between the GC-indices of upwards and downwards direction (B-C and G-H, blue). The brightness

682 of colors indicate the size of the stimulus (dark: large, light: small). 

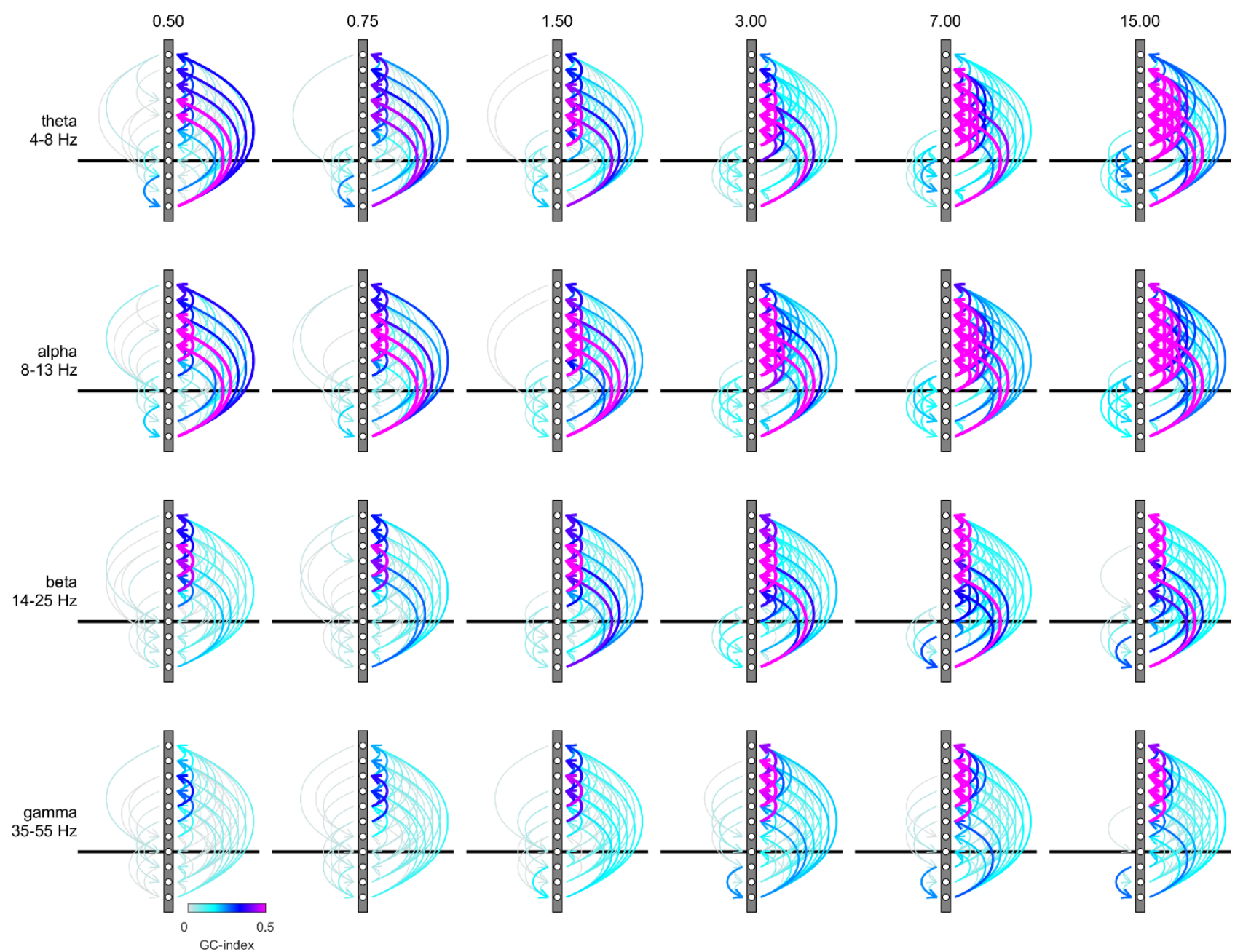

685 Figure S6: Connection plots of net Granger-causality in four frequency bands. Conventions are the

686 same as in figure 7 in the main text. The figure shows the raw GC-indices without testing the

687 directionality with the reverse-granger-test. 

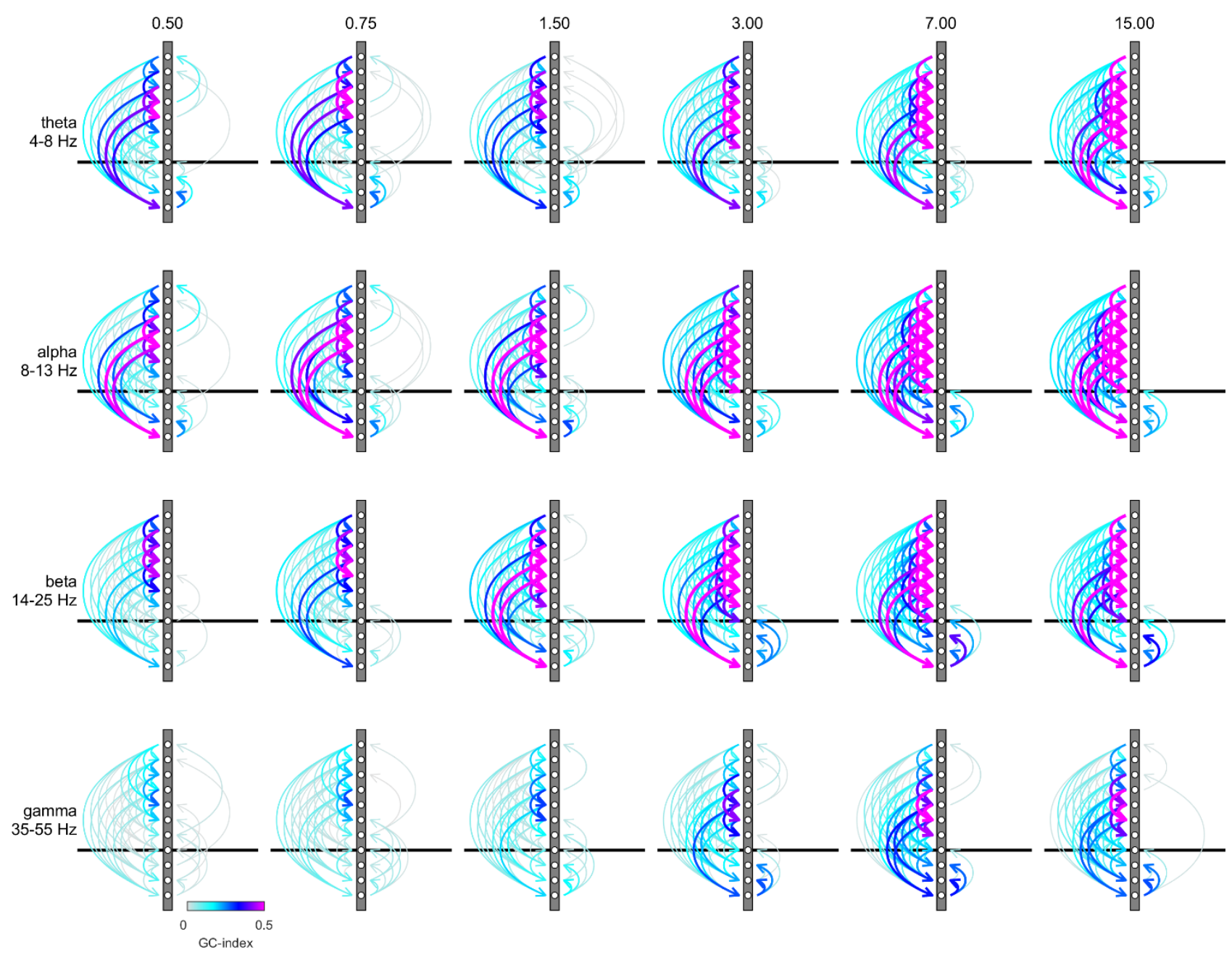

690 Figure S7: Connection plots of net Reverse Granger-causality in four frequency bands. Conventions

691 are the same as in figure 7 in the main text, but the figure shows the results of the reverse-granger-

692 test, i.e. the net Granger-causality calculated with time reversal. 